\title{
Relativistic pionic effects in quasielastic electron scattering
}

\author{
J.E. Amaro ${ }^{1}$, M.B. Barbaro ${ }^{2}$, J.A. Caballero ${ }^{3,4}$, T.W. Donnelly ${ }^{5}$ and A. \\ Molinari $^{2}$ \\ ${ }^{1}$ Departamento de Física Moderna, Universidad de Granada, E-18071 Granada, SPAIN \\ ${ }^{2}$ Dipartimento di Fisica Teorica, Università di Torino and INFN, Sezione di Torino \\ Via P. Giuria 1, 10125 Torino, ITALY \\ ${ }^{3}$ Departamento de Física Atómica, Molecular y Nuclear \\ Universidad de Sevilla, Apdo. 1065, E-41080 Sevilla, SPAIN \\ ${ }^{4}$ Instituto de Estructura de la Materia, CSIC Serrano 123, E-28006 Madrid, SPAIN \\ ${ }^{5}$ Center for Theoretical Physics, Laboratory for Nuclear Science and Department of Physics \\ Massachusetts Institute of Technology, Cambridge, MA 02139, USA
}

\begin{abstract}
The impact of relativistic pionic correlations and meson-exchange currents on the response functions for electromagnetic quasielastic electron scattering from nuclei is studied in detail. Results in first-order perturbation theory are obtained for one-particle emission electronuclear reactions within the context of the relativistic Fermi gas model. Improving upon previous analyses where non-relativistic reductions of the currents were performed, here a fully relativistic analysis in which both forces and currents are treated consistently is presented. Lorentz covariance is shown to play a crucial role in enforcing the gauge invariance of the theory. Effects stemming uniquely from relativity in the pionic correlations are identified and, in particular, a comprehensive study of the self-energy contributions and of the currents associated with the pion is presented. First- and second-kind scaling for high momentum transfer is investigated.
\end{abstract}

PACS: 25.30.Rw, 14.20.Gk, 24.10.Jv, 24.30.Gd, 13.40.Gp Keywords: Nuclear reactions; Inclusive electron scattering; Pionic correlations. Meson-exchange currents. Relativistic Fermi Gas.

MIT/CTP\#3127

May 31, 2001 


\section{Introduction}

For years electron scattering has provided one of the most powerful means to study the structure of nuclei. In particular, inclusive $\left(\mathrm{e}, \mathrm{e}^{\prime}\right)$ processes at or near quasielastic peak kinematics have attracted attention in the last two decades and a number of experiments have been performed with the aim of disentangling the longitudinal and transverse contributions to the quasielastic cross section. Calculations based on a simple picture of the dynamics of the quasielastic peak (one-photon-exchange, impulse approximation and one-body currents) appeared to be successful in explaining the early $\left(\mathrm{e}, \mathrm{e}^{\prime}\right)$ cross section data for several nuclei [1], except at large energy transfer where $\Delta$ excitation of the nucleon contributes substantially. However, a simultaneous explanation of the separated longitudinal, $R^{L}$, and transverse, $R^{T}$, response functions could not be fully reached when representing the quasifree peak simply as an incoherent sum of elastic nucleon scattering processes. Some authors attributed this problem to a large quenching thought to occur in $R^{L}$ [2, 3, 4, 5], although others found no strong evidence for such a quenching [6, 7, 8] and later analysis of the world data [9] showed that the reduction of the longitudinal response at high momentum transfers was actually smaller than previously assumed. Thus at present it appears more likely that the "quenching" of the longitudinal response is not where the problem lies, but rather that the transverse response has contributions other than those mentioned above - some of these contributions constitute the focus of the present work.

Different approaches to this problem have been invoked. As mentioned above, the earlier approaches used only one-body (nucleonic) currents, i.e., the impulse approximation (IA). Whereas the IA explains the electron scattering reaction mechanism around the quasielastic peak reasonably well, theoretical models based on one-body currents are unable to account for the observed strength in the dip region between the quasielastic and $\Delta$ peaks, where meson production, including via the $\Delta$, and two-body currents [10] should be taken into account in the analysis. Several studies dealing with the issue of two-body currents in quasielastic electron scattering reactions have been published. Most focused on the role played by the meson-exchange currents (MEC), specifically the seagull and pion-in-flight

terms. For instance, detailed results were obtained with the Fermi gas model (RFG) [11, 12, 13] and, as well, using a continuum shell model [14, 15, 16]. Moreover, the contribution of the $\Delta$ isobar current, i.e., the current associated with the electromagnetic excitation of an intermediate isobar, which decays by exchanging a virtual pion with a nucleon, has been also considered [12, 16, 17] and shown, in most cases, to be larger than the purely $\pi N$ MEC contributions.

In some previous work [18] a relativistic analysis of the MEC has been presented (although there the associated correlations were neglected and thus gauge invariance was not respected); however, most calculations have been done basically within a non-relativistic framework. As a consequence, not only have non-relativistic wave functions been used, but also nonrelativistic current operators, the latter being obtained using standard expansions which view both the dimensionless momentum transfer $\kappa \equiv q / 2 m$ and the dimensionless energy transfer $\lambda \equiv \omega / 2 m$ as being small ( $m$ is the nucleon mass). Obviously, for high-energy conditions, the current operators so obtained are inadequate and new expressions are needed. This provided the focus for the developments in [19] where new current operators that are exact as far as 
the variables $\omega$ and $q$ are concerned were derived.

As mentioned above, another difficulty of most of the non-relativistic pionic calculations existing in the literature relates to the breakdown of gauge invariance. In this connection, we recall some differences between the pionic and isobar two-body current operators. In a representation of the nucleus with states described only in terms of nucleons and where current and interaction effects are embodied in operators that contain all other hadronic degrees of freedom (pions, deltas, ...) these two enter somewhat differently. In lowest order the latter is gauge invariant by itself, whereas the former enters both in one-boson-exchange interactions (i.e., potentials in the non-relativistic limit) and in one-boson-exchange currents. Thus the two-body pionic currents must, via the continuity equation, be connected with the interaction effects, the so-called correlations. One might expect such pionic contributions to be more constrained than those arising from the isobar. But in most non-relativistic calculations the contribution of $\pi$ meson-exchange currents violates current conservation. This constitutes a fundamental flaw for the consistency of the theory and hence is one of the main issues addressed in this work.

In this paper our aim is to investigate the role of pions in inclusive electron scattering from nuclei within the kinematical regime of the quasielastic peak. We restrict our attention to the study of the purely electromagnetic response functions, $R^{L}$ and $R^{T}$, leaving for subsequent presentation the analysis of parity-violating responses and of the roles played in the responses by mesons heavier than the pion. Furthermore, here our focus is placed on the contributions that affect the one-particle one-hole $(1 \mathrm{p}-1 \mathrm{~h})$ sector of the nuclear excitations, whereas in the near future we shall also present results within the same framework for contributions that affect the $2 \mathrm{p}-2 \mathrm{~h}$ excitations.

This work extends the approach presented in a previous paper [12], but introduces some important new elements, not only in connection with the implementation of Lorentz covariance, but also as far as the self-consistency of the theory (specifically the fulfillment of gauge invariance) is concerned. As in [12] we undertake this study within the context of the RFG model, which is well suited for maintaining the consistency in the treatment of the forces and currents while fullfilling the Lorentz covariance. The RFG is in fact the simplest model that provides a valid starting framework for describing quasielastic electron scattering and exactly respects relativity. To employ this model and to extend it using standard perturbation theory to account for first-order pionic effects amounts to considering all Feynman diagrams carrying one pionic line (a discussion of the relevance of higher-order pionic diagrams can be found in [20, 21]). Accordingly, our theory is based on these diagrams and we do not make any non-relativistic expansion when handling the pionic current operators; herein lies the main difference with previous work [12, 19, 22] where some kind of expansion in powers of $1 / m$ was introduced. In the present study we present a fully relativistic analysis including the pionic correlation effects, embodied in the so-called self-energy and exchange diagrams, as well as the MEC contributions (seagull and pion-in-flight terms). The analysis of the $\Delta$ current, within the same framework, will be presented in a forthcoming publication.

With regard to gauge invariance, in contrast to previous (non-relativistic) analyses, our findings strongly support the need for a fully relativistic treatment in order to preserve this symmetry. In addition, relativity is clearly manifested in new effects which appear at the level of the currents and/or propagators and which are absent in the non-relativistic approxi- 
mations. The relativistic analysis of the self-energy (s.e.) term that presents some important differences compared with the non-relativistic approach is particularly significant. This issue will be treated in detail in forthcoming work [23] and so here we only mention the fact that two different contributions in the s.e. pionic correlation term can be identified. One stems from the renormalization of the energy and the other has its origin in the lower components of the Dirac wave functions. Whereas the first term can be shown to correspond to the one already embodied in the s.e. diagram within the non-relativistic approach [12], the second one has no counterpart in the non-relativistic analysis. However, both contributions are necessary, not only to set up a consistent and well-defined self-energy particle-hole (ph) current matrix element, but also to fulfill the continuity equation expressing the gauge invariance of the theory.

This paper is organized as follows: in Section 2 we present the general formalism and the basic ingredients that enter in analyses of inclusive quasielastic electron scattering. We define the longitudinal and transverse electromagnetic inclusive response functions in terms of the hadronic tensor $W^{\mu \nu}$. This in turn can be constructed through the ph current matrix elements or, equivalently, as the imaginary part of the polarization propagator. Section 3 deals with the one-pion-exchange contributions to the nuclear responses. We start from the Feynman diagrams corresponding to the free-space MEC and pion induced nucleon-nucleon correlation currents. We prove, at this level, the gauge invariance of the theory. From these currents we next set up the ph matrix elements that enter in our RFG-based computation. The case of the s.e. diagram is discussed in detail as the corresponding ph current matrix element is divergent. We treat this term using the polarization propagator approach, thus obtaining a finite response. In [23] we shall prove, however, that it is possible to derive a new "renormalized" expression for the self-energy current matrix element which is finite, consistent with the analysis performed with the polarization propagator technique and, when taken together with the remaining pionic currents and the one-body current, satisfies current conservation. In Section 4 we discuss the effects introduced by the MEC and pion correlations at the level of the two response functions, $R^{L}$ and $R^{T}$. We study the magnitude of these effects as a function of the transfer momentum $q$, and, as well, their dependence upon the Fermi momentum $k_{F}$. Such dependences are clearly relevant in connection with analyses of scaling in nuclei [24], both of first and second kinds, and, as we shall see, scaling of the former kind is attained at high $q$, whereas scaling of the latter kind is clearly broken through dependence roughly on the Fermi momentum squared for all values of $q$. The issue of the impact on the responses of the dynamical pion propagator, versus the standard static propagator, is also discussed. Finally, in Sections 5 and 6 we summarize our results and draw our conclusions.

\section{General Formalism}

In this section we briefly summarize the general formalism involved in the description of $\left(\mathrm{e}, \mathrm{e}^{\prime}\right)$ processes for quasielastic kinematics. We only discuss those aspects that are of special relevance to the analysis that follows and the reader who is interested in more details will benefit from reading [25, 26, 27, 28, 29]. We limit our attention to the Plane Wave Born 
Approximation (PWBA) in which the electron is described as a plane wave and interacts with the nuclear target via the exchange of a virtual photon. The variables involved in the process are $K_{e}^{\mu}=(\varepsilon, \mathbf{k})$ and $K_{e}^{\prime \mu}=\left(\varepsilon^{\prime}, \mathbf{k}^{\prime}\right)$, the initial and scattered electron four-momenta, and $P_{i}^{\mu}=$ $\left(M_{i}, \mathbf{0}\right)$ and $P_{f}^{\mu}=\left(E_{f}, \mathbf{p}_{f}\right)$, the initial and final hadronic four-momenta, respectively. The four-momentum transferred by the virtual photon is $Q^{\mu}=\left(K_{e}-K_{e}^{\prime}\right)^{\mu}=\left(P_{f}-P_{i}\right)^{\mu}=(\omega, \mathbf{q})$. Assuming Lorentz invariance and parity conservation, the inclusive $\left(\mathrm{e}, \mathrm{e}^{\prime}\right)$ cross section then reads

$$
\frac{d \sigma}{d \Omega_{e}^{\prime} d \omega}=\frac{2 \alpha^{2}}{Q^{4}}\left(\frac{\varepsilon^{\prime}}{\varepsilon}\right) \eta_{\mu \nu} W^{\mu \nu}=\sigma_{M}\left[v_{L} R^{L}(q, \omega)+v_{T} R^{T}(q, \omega)\right],
$$

where $\alpha$ is the fine structure constant, $\sigma_{M}$ the Mott cross section and $\Omega_{e}^{\prime}$ the scattered electron solid angle. The terms $\eta_{\mu \nu}$ and $W^{\mu \nu}$ represent the leptonic and hadronic tensors, respectively. The kinematic factors $v_{L}$ and $v_{T}$ are evaluated from the leptonic tensor with standard techniques (see [25] for explicit expressions), whereas the longitudinal and transverse (with respect to the momentum transfer $\mathbf{q}$ ) response functions $R^{L}$ and $R^{T}$ are constructed directly as components of the hadronic tensor $W^{\mu \nu}$ according to

$$
\begin{aligned}
& R^{L}(q, \omega)=\left(\frac{q^{2}}{Q^{2}}\right)^{2}\left[W^{00}-\frac{\omega}{q}\left(W^{03}+W^{30}\right)+\frac{\omega^{2}}{q^{2}} W^{33}\right] \\
& R^{T}(q, \omega)=W^{11}+W^{22} .
\end{aligned}
$$

Note that if gauge invariance is fulfilled, implying that $W^{03}=W^{30}=(\omega / q) W^{00}$ and $W^{33}=(\omega / q)^{2} W^{00}$, then $R^{L}$ is simply the time component of the hadronic tensor, namely $W^{00}$. Hence $R^{L}$ is determined by the charge distribution, whereas $R^{T}$ reflects the current distribution of the nuclear target.

The hadronic tensor and consequently the response functions derived from it embody the entire dependence on the nuclear structure, specifically on the charge and current distributions in nuclei, and accordingly these provide the prime focus in analyses of electron scattering. There are various options on how to proceed in performing such analyses (see, for example, [30]), depending on the specific problem under consideration and on the approximations to be made. In what follows we recall two common expressions for the hadronic tensor $W^{\mu \nu}$ and comment briefly on their applications.

First, the hadronic tensor can be defined according to

$$
W^{\mu \nu}=\overline{\sum_{i}} \sum_{f}\left\langle f\left|\hat{J}^{\mu}\right| i\right\rangle^{*}\left\langle f\left|\hat{J}^{\nu}\right| i\right\rangle \delta\left(E_{i}+\omega-E_{f}\right),
$$

where $\hat{J}^{\mu}$ represents the nuclear many-body current operator, the nuclear states $|i\rangle$ and $|f\rangle$ are exact eigenstates of the nuclear Hamiltonian with definite four-momentum, and the sum with a bar means average over initial states. This form is very general and includes all possible final states that can be reached through the action of the current operator $\hat{J}^{\mu}$ on the exact ground state. In our perturbative approach we shall use eigenstates of the free Hamiltonian $H_{0}$ (which describes the free RFG) and include correlations among nucleons in the current mediated by the exchange of pions (MEC). This current of course allows one to 
reach both the $\mathrm{p}-\mathrm{h}$ and the $2 \mathrm{p}-2 \mathrm{~h}$ sectors in the Hilbert space of $H_{0}$. In the present work, however, we shall restrict our attention to the former.

A different option for evaluating the nuclear responses exploits the polarization propagator $\Pi^{\mu \nu}$ (also referred to as the current-current correlation function). The latter can be

expressed in terms of the full propagator, $\hat{G}$, of the nuclear many-body system, since closure can be used to carry out the sum over the final states in eq. (代. Then one has for the hadronic tensor

$$
W^{\mu \nu}=-\frac{1}{\pi} \operatorname{Im}\left(\Pi^{\mu \nu}\right)=-\frac{1}{\pi} \operatorname{Im}\left(\overline{\sum_{i}}\left\langle i\left|\hat{J}^{\dagger \mu} \hat{G}\left(\omega+E_{i}\right) \hat{J}^{\nu}\right| i\right\rangle\right) .
$$

A possible advantage of this approach relates to the existence of a well-defined set of rules (the relativistic Feynman diagrams) which allows one to compute $\Pi^{\mu \nu}$ perturbatively [31.

Obviously both procedures are equivalent and hence the observables calculated using the expressions for the hadronic tensor given by eqs. (4) or (5) should be the same. However, notice that eq. (4) is less suitable for dealing with situations where the nuclear current matrix element $\left\langle f\left|\hat{J}^{\mu}\right| i\right\rangle$ is divergent. In this case it is advisable to compute the responses via the polarization propagator.

Finally, we remark that gauge invariance must be fulfilled both at the level of the nuclear current matrix elements and at the level of the hadronic tensor and/or the polarization propagator. A consequence is that the electromagnetic continuity equation should be satisfied. In other words in momentum space all of the expressions $Q_{\mu}\left\langle f\left|\hat{J}^{\mu}\right| i\right\rangle, Q_{\mu} W^{\mu \nu}$ and $Q_{\mu} \Pi^{\mu \nu}$ should vanish.

\section{Pion exchange contributions}

In this Section we shall discuss the hadronic tensor and response functions in the framework of the RFG model, i.e. for nucleons moving freely inside the nucleus with relativistic kinematics, accounting for the effects introduced by pions in first-order perturbation theory (one-pionexchange). We shall employ pseudovector (PV) coupling for the pion-nucleon interactions.

\subsection{Feynman diagrams and two-body currents}

We start by displaying in Fig. 1 the Feynman diagrams corresponding to the free-space two-body currents with only one-pion-exchange. Using the Feynman rules one writes down the corresponding expressions for the two-particle current matrix elements. Following standard terminology, diagrams (a), (b) and (c) correspond to the usual meson-exchange currents where the electromagnetic field is coupled with the pionic current: in particular, diagrams (a) and (b) are referred to as the seagull (contact) term and (c) as the pion-in-flight term, respectively. Diagrams (d)-(g) contain an intermediate virtual nucleon and give rise to the so-called correlation currents. In some work [32] it has been argued that the contribution of these "correlation" diagrams is already contained in the nuclear wave functions as solutions of the Schrödinger equation. However, within the RFG plus first-order perturbation theory framework the unperturbed basis does not have any such contributions and so within this 


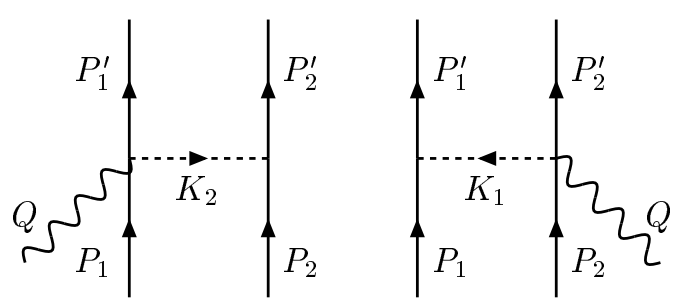

(a) (b)

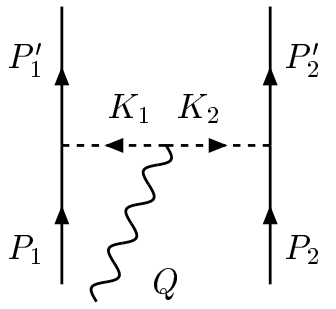

(c)

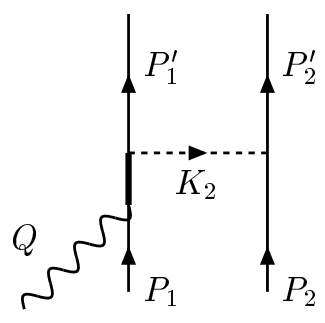

(d)

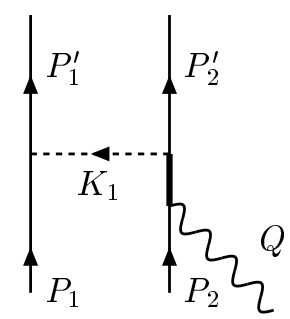

(e)

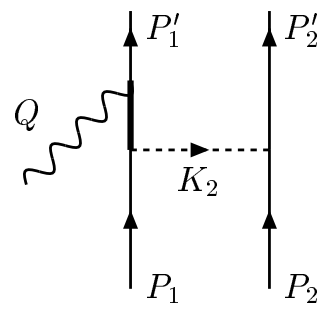

(f)

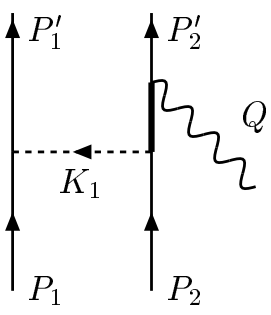

(g)

Figure 1: Free-space two-body current diagrams: Seagull (a,b), pion-in-flight (c) and correlation (d-g) contributions.

approach they must be included explicitly — in fact, as we shall see, their inclusion is crucial for fulfilling gauge invariance.

The relativistic expressions for the free-space two-particle currents are (isospin summations are understood)

- Seagull or contact:

$$
\begin{aligned}
j_{\mu}^{S}(Q) & =\left(\frac{f m}{V m_{\pi}}\right)^{2} \frac{1}{\sqrt{E_{\mathbf{p}_{1}} E_{\mathbf{p}_{2}} E_{\mathbf{p}_{1}^{\prime}} E_{\mathbf{p}_{2}^{\prime}}}} i \epsilon_{3 a b} \bar{u}\left(\mathbf{p}_{1}^{\prime}\right) \tau_{a} \gamma_{5} K_{1} u\left(\mathbf{p}_{1}\right) \frac{F_{1}^{V}}{K_{1}^{2}-m_{\pi}^{2}} \bar{u}\left(\mathbf{p}_{2}^{\prime}\right) \tau_{b} \gamma_{5} \gamma_{\mu} u\left(\mathbf{p}_{2}\right) \\
& +(1 \leftrightarrow 2)
\end{aligned}
$$

- Pion-in-flight:

$$
\begin{aligned}
j_{\mu}^{P}(Q) & =\left(\frac{f m}{V m_{\pi}}\right)^{2} \frac{1}{\sqrt{E_{\mathbf{p}_{1}} E_{\mathbf{p}_{2}} E_{\mathbf{p}_{1}^{\prime}} E_{\mathbf{p}_{2}^{\prime}}}} i \epsilon_{3 a b} \frac{F_{\pi}\left(K_{1}-K_{2}\right)_{\mu}}{\left(K_{1}^{2}-m_{\pi}^{2}\right)\left(K_{2}^{2}-m_{\pi}^{2}\right)} \\
& \times \bar{u}\left(\mathbf{p}_{1}^{\prime}\right) \tau_{a} \gamma_{5} \not K_{1} u\left(\mathbf{p}_{1}\right) \bar{u}\left(\mathbf{p}_{2}^{\prime}\right) \tau_{b} \gamma_{5} \not K_{2} u\left(\mathbf{p}_{2}\right)
\end{aligned}
$$

- Correlation: 


$$
\begin{aligned}
j_{\mu}^{C}(Q)= & \left(\frac{f m}{V m_{\pi}}\right)^{2} \frac{1}{\sqrt{E_{\mathbf{p}_{1}} E_{\mathbf{p}_{2}} E_{\mathbf{p}_{1}^{\prime}} E_{\mathbf{p}_{2}^{\prime}}}} \bar{u}\left(\mathbf{p}_{1}^{\prime}\right) \tau_{a} \gamma_{5} \not K_{1} u\left(\mathbf{p}_{1}\right) \frac{1}{K_{1}^{2}-m_{\pi}^{2}} \\
& \times \bar{u}\left(\mathbf{p}_{2}^{\prime}\right)\left[\tau_{a} \gamma_{5} K_{1} S_{F}\left(P_{2}+Q\right) \Gamma^{\mu}(Q)+\Gamma^{\mu}(Q) S_{F}\left(P_{2}^{\prime}-Q\right) \tau_{a} \gamma_{5} \not K_{1}\right] u\left(\mathbf{p}_{2}\right) \\
& +(1 \leftrightarrow 2) .
\end{aligned}
$$

In the above, $V$ is the volume enclosing the system, $m_{\pi}$ is the mass of the pion, $f$ the pion-nucleon coupling constant and $E_{\mathbf{p}}=\sqrt{\mathbf{p}^{2}+m^{2}}$ the on-shell energy of a nucleon with momentum p; the four-momenta - indicated by capital letters - are defined in Fig. 1 and $F_{1}^{V}$ and $F_{\pi}$ are the electromagnetic isovector nucleon and pion form factors, respectively. Furthermore, $S_{F}(P)$ is the nucleon propagator and $\Gamma^{\mu}(Q)$ the electromagnetic nucleon vertex, i.e.,

$$
\begin{aligned}
S_{F}(P) & =\frac{P+m}{P^{2}-m^{2}} \\
\Gamma^{\mu}(Q) & =F_{1} \gamma^{\mu}+\frac{i}{2 m} F_{2} \sigma^{\mu \nu} Q_{\nu},
\end{aligned}
$$

$F_{1}$ and $F_{2}$ being the Dirac and Pauli form factors: for these we use the Galster parameterization [33]. Finally, the spinors (for brevity we denote $u\left(\mathbf{p}, s_{p}\right)$ by $u(\mathbf{p})$ ) are normalized according to the Bjorken and Drell convention [34 and isospinors are not explicitly indicated.

The seagull and pion-in-flight currents shown above coincide with the expressions given by Van Orden and Donnelly [11] if account is taken for the different conventions used for the gamma matrix $\gamma_{5}$ and for the metric. Concerning the correlation current, note that, at variance with [11, it embodies both the positive and negative energy components of the nucleon propagator.

An important point to be stressed is that the relativistic seagull, pion-in-flight and correlation currents satisfy current conservation, i.e. $Q_{\mu} J^{\mu}=0$, provided some assumptions are made for the form factors involved in the various currents. To prove this statement we start by evaluating the contraction of the four-momentum transfer $Q^{\mu}$ with the correlation current $j_{\mu}^{C}$. It can be written as

$$
Q^{\mu} j_{\mu}^{C}=\left(\frac{f m}{V m_{\pi}}\right)^{2} \frac{1}{\sqrt{E_{\mathbf{p}_{1}} E_{\mathbf{p}_{2}} E_{\mathbf{p}_{1}^{\prime}} E_{\mathbf{p}_{2}^{\prime}}}} \bar{u}\left(\mathbf{p}_{1}^{\prime}\right) \tau_{a} \gamma_{5} \not K_{1} u\left(\mathbf{p}_{1}\right) \frac{1}{K_{1}^{2}-m_{\pi}^{2}} \mathcal{M}_{a}+(1 \leftrightarrow 2)
$$

with $\mathcal{M}_{a}$ given by

$$
\mathcal{M}_{a}=\bar{u}\left(\mathbf{p}_{2}^{\prime}\right)\left[\tau_{a} \gamma_{5} K_{1} S_{F}\left(P_{2}+Q\right) \varnothing F_{1}+F_{1} \varnothing S_{F}\left(P_{2}^{\prime}-Q\right) \tau_{a} \gamma_{5} K_{1}\right] u\left(\mathbf{p}_{2}\right),
$$

where we have used the relation $Q^{\mu} \Gamma_{\mu}(Q)=F_{1}(Q)$ Q. After some algebra, involving the nucleon propagator and the Dirac spinors, $\mathcal{M}_{a}$ can be further simplified leading to the form

$$
\mathcal{M}_{a}=\bar{u}\left(\mathbf{p}_{2}^{\prime}\right)\left[\tau_{a} \gamma_{5} K_{1} F_{1}-F_{1} \tau_{a} \gamma_{5} K_{1}\right] u\left(\mathbf{p}_{2}\right)=\bar{u}\left(\mathbf{p}_{2}^{\prime}\right)\left[\tau_{a}, F_{1}\right] \gamma_{5} K_{1} u\left(\mathbf{p}_{2}\right) .
$$

In order to evaluate the commutator $\left[\tau_{a}, F_{1}\right]$ we decompose the nucleon form factor into its isoscalar and isovector pieces, $F_{1}=\frac{1}{2}\left(F_{1}^{S}+F_{1}^{V} \tau_{3}\right)$. Then

$$
\left[\tau_{a}, F_{1}\right]=-i F_{1}^{V} \epsilon_{3 a b} \tau_{b},
$$


which entails the automatic conservation of the $\pi^{0}$ exchange current $(a=3)$. Using eq. (14) we can recast $\mathcal{M}_{a}$ as follows

$$
\mathcal{M}_{a}=-i F_{1}^{V} \epsilon_{3 a b} \bar{u}\left(\mathbf{p}_{2}^{\prime}\right) \tau_{b} \gamma_{5} K_{1} u\left(\mathbf{p}_{2}\right) ;
$$

hence the divergence of the two-body correlation current matrix element can finally be written as

$$
\begin{aligned}
Q^{\mu} j_{\mu}^{C} & =2 m\left(\frac{f m}{V m_{\pi}}\right)^{2} \frac{1}{\sqrt{E_{\mathbf{p}_{1}} E_{\mathbf{p}_{2}} E_{\mathbf{p}_{1}^{\prime}} E_{\mathbf{p}_{2}^{\prime}}}} i \epsilon_{3 a b} \bar{u}\left(\mathbf{p}_{1}^{\prime}\right) \tau_{a} \gamma_{5} u\left(\mathbf{p}_{1}\right) \frac{F_{1}^{V}}{K_{1}^{2}-m_{\pi}^{2}} \\
& \times \bar{u}\left(\mathbf{p}_{2}^{\prime}\right) \tau_{b} \gamma_{5}(\varnothing+2 m) u\left(\mathbf{p}_{2}\right)+(1 \leftrightarrow 2) .
\end{aligned}
$$

The divergence of the seagull and pion-in-flight two-body current matrix elements can also be calculated in a straightforward way. The final result reads

$$
\begin{aligned}
Q^{\mu} j_{\mu}^{S} & =-2 m\left(\frac{f m}{V m_{\pi}}\right)^{2} \frac{1}{\sqrt{E_{\mathbf{p}_{1}} E_{\mathbf{p}_{2}} E_{\mathbf{p}_{1}^{\prime}} E_{\mathbf{p}_{2}^{\prime}}}} i \epsilon_{3 a b} \bar{u}\left(\mathbf{p}_{1}^{\prime}\right) \tau_{a} \gamma_{5} u\left(\mathbf{p}_{1}\right) \frac{F_{1}^{V}}{K_{1}^{2}-m_{\pi}^{2}} \\
& \times \bar{u}\left(\mathbf{p}_{2}^{\prime}\right) \tau_{b} \gamma_{5} \emptyset u\left(\mathbf{p}_{2}\right)+(1 \leftrightarrow 2) \\
Q^{\mu} j_{\mu}^{P} & =4 m^{2}\left(\frac{f m}{V m_{\pi}}\right)^{2} \frac{1}{\sqrt{E_{\mathbf{p}_{1}} E_{\mathbf{p}_{2}} E_{\mathbf{p}_{1}^{\prime}} E_{\mathbf{p}_{2}^{\prime}}}} i \epsilon_{3 a b} F_{\pi} \frac{\left(K_{1}-K_{2}\right) \cdot Q}{\left(K_{1}^{2}-m_{\pi}^{2}\right)\left(K_{2}^{2}-m_{\pi}^{2}\right)} \\
& \times \bar{u}\left(\mathbf{p}_{1}^{\prime}\right) \tau_{a} \gamma_{5} u\left(\mathbf{p}_{1}\right) \bar{u}\left(\mathbf{p}_{2}^{\prime}\right) \tau_{b} \gamma_{5} u\left(\mathbf{p}_{2}\right) .
\end{aligned}
$$

Then, by summing up the contributions given by the correlation (eq. (16)) and seagull (eq. (17)) currents and writing the four-momentum transfer as $Q_{\mu}=\left(K_{1}+K_{2}\right)_{\mu}$, we finally obtain

$$
\begin{aligned}
Q^{\mu}\left(j_{\mu}^{C}+j_{\mu}^{S}\right) & =4 m^{2}\left(\frac{f m}{V m_{\pi}}\right)^{2} \frac{1}{\sqrt{E_{\mathbf{p}_{1}} E_{\mathbf{p}_{2}} E_{\mathbf{p}_{1}^{\prime}} E_{\mathbf{p}_{2}^{\prime}}}} F_{1}^{V} i \epsilon_{3 a b} \frac{\left(K_{2}-K_{1}\right) \cdot Q}{\left(K_{1}^{2}-m_{\pi}^{2}\right)\left(K_{2}^{2}-m_{\pi}^{2}\right)} \\
& \times \bar{u}\left(\mathbf{p}_{1}^{\prime}\right) \tau_{a} \gamma_{5} u\left(\mathbf{p}_{1}\right) \bar{u}\left(\mathbf{p}_{2}^{\prime}\right) \tau_{b} \gamma_{5} u\left(\mathbf{p}_{2}\right)
\end{aligned}
$$

which cancels exactly the contribution of pion-in-flight current in eq. (18) provided the electromagnetic pion form factor is chosen to be $F_{\pi}=F_{1}^{V}$, which we shall assume in this paper.

We have thus proven that the sum of the relativistic two-body matrix elements of the MEC and of the correlation currents satisfies the continuity equation if the same electromagnetic form factors enter in all of the currents: namely one has $Q^{\mu}\left(j_{\mu}^{C}+j_{\mu}^{S}+j_{\mu}^{P}\right)=0$.

\subsection{Particle-hole matrix elements}

In this paper we focus on the one-particle emission reactions within the framework of the RFG. Therefore, one has to evaluate the matrix element of the two-body current operators taken between the Fermi sphere and a particle-hole state, namely

$$
\left\langle p h^{-1}\left|\hat{j}_{\mu}\right| F\right\rangle=\sum_{s_{k}, t_{k}} \sum_{\mathbf{k} \leq k_{F}}\left[\left\langle p k\left|\hat{j}_{\mu}\right| h k\right\rangle-\left\langle p k\left|\hat{j}_{\mu}\right| k h\right\rangle\right]
$$




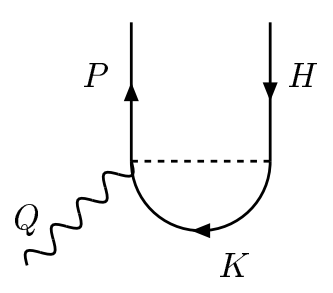

(a)

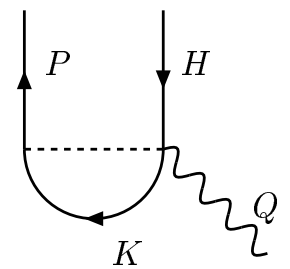

(b)

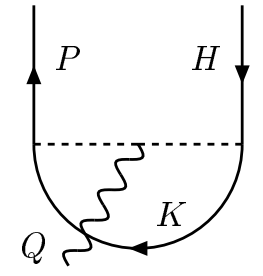

(c)

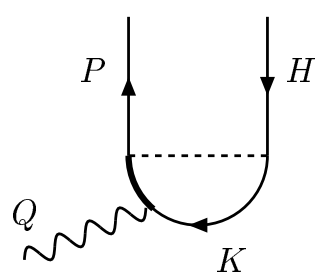

(d)

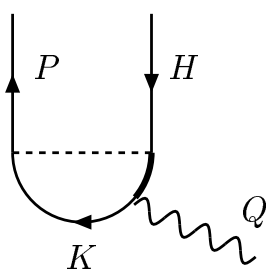

(e)

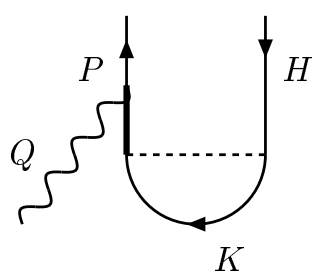

(f)

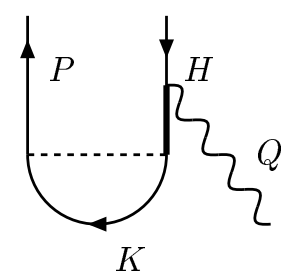

$(\mathrm{g})$

Figure 2: Feynman diagrams representing the seagull (a and b), pion-in-flight (c), vertex (d and e) and self-energy of the hole (f) and particle (g) particle-hole matrix elements.

where the sum $\sum_{\mathbf{k} \leq k_{F}}$ in the thermodynamic limit becomes an integral over the momentum in the range $0 \leq k \leq k_{F}$, with $k_{F}$ the Fermi momentum, and over the angular variables $\theta_{k}, \phi_{k}$. The first and second terms in eq. (20) represent the direct and exchange contribution to the matrix element, respectively. It is well-known that the direct term vanishes for the MEC (seagull and pion-in-flight) currents (see [19]) because of both spin-isospin traces (in spin-isospin saturated systems) and the presence of a pion carrying zero momentum. It is easily verified that, for the same reasons, the direct matrix element of the correlation current also does not contribute to the responses.

Hence only the exchange term remains in the particle-hole matrix elements for the twobody current operators. The corresponding Feynman diagrams are displayed in Fig. 2. Diagrams (a-b) and (c) correspond to the seagull (contact) and pion-in-flight terms, respectively. Diagrams (d-e) represent instead the correlation contributions. Here we distinguish the self-energy insertions on the nucleonic lines ( $\mathrm{f}, \mathrm{g})$ and the exchange of a pion between a particle and a hole line $(\mathrm{d}, \mathrm{e})$.

Starting from the general expressions in eqs. (6, 7,8) we can now evaluate the particle-hole matrix elements. Carrying out explicitly the sums over the spin, $s_{k}$, and isospin, $t_{k}$, variables and after some laborious, but straightforward, algebra we finally get

- Seagull 


$$
\begin{aligned}
& \left\langle p h^{-1}\left|\hat{j}_{\mu}^{S}\right| F\right\rangle=-\frac{m f^{2}}{V^{2} m_{\pi}^{2} \sqrt{E_{\mathbf{p}} E_{\mathbf{h}}}} F_{1}^{(V)} i \varepsilon_{3 a b} \\
& \quad \times \sum_{\mathbf{k}} \frac{m}{E_{\mathbf{k}}} \bar{u}(\mathbf{p}) \tau_{a} \tau_{b}\left\{\frac{(K-m) \gamma_{\mu}}{(P-K)^{2}-m_{\pi}^{2}}+\frac{\gamma_{\mu}(K-m)}{(K-H)^{2}-m_{\pi}^{2}}\right\} u(\mathbf{h})
\end{aligned}
$$

- Pion-in-flight

$$
\begin{aligned}
& \left\langle p h^{-1}\left|\hat{j}_{\mu}^{P}\right| F\right\rangle=2 m \frac{m f^{2}}{V^{2} m_{\pi}^{2} \sqrt{E_{\mathbf{p}} E_{\mathbf{h}}}} F_{1}^{(V)} i \varepsilon_{3 a b} \\
& \quad \times \sum_{\mathbf{k}} \frac{m}{E_{\mathbf{k}}} \frac{(Q+2 H-2 K)_{\mu}}{\left[(P-K)^{2}-m_{\pi}^{2}\right]\left[(K-H)^{2}-m_{\pi}^{2}\right]} \bar{u}(\mathbf{p}) \tau_{a}(K-m) \tau_{b} u(\mathbf{h})
\end{aligned}
$$

- Correlation

$$
\begin{aligned}
& \left\langle p h^{-1}\left|\hat{j}_{\mu}^{C}\right| F\right\rangle=-\frac{1}{2 m} \frac{m f^{2}}{V^{2} m_{\pi}^{2} \sqrt{E_{\mathbf{p}} E_{\mathbf{h}}}} \sum_{\mathbf{k}} \frac{m}{E_{\mathbf{k}}} \bar{u}(\mathbf{p})\left\{\tau_{a} \gamma_{5} \frac{\not P-\not K}{(P-K)^{2}-m_{\pi}^{2}}(K+m)\right. \\
& \times\left[\tau_{a} \gamma_{5}(P-\not K) S_{F}(H+Q) \Gamma_{\mu}(Q)+\Gamma_{\mu}(Q) S_{F}(K-Q) \tau_{a} \gamma_{5}(P-\not K)\right] \\
& +\left[\tau_{a} \gamma_{5}(K-\not H) S_{F}(K+Q) \Gamma_{\mu}(Q)+\Gamma_{\mu}(Q) S_{F}(P-Q) \tau_{a} \gamma_{5}(K-\not H)\right] \\
& \left.\times(K+m) \tau_{a} \gamma_{5} \frac{\not K-\not H}{(K-H)^{2}-m_{\pi}^{2}}\right\} u(\mathbf{h}) .
\end{aligned}
$$

The first and last terms in eq. (23) represent the so-called self-energy (s.e.) matrix elements (diagrams (g) and (f) in Fig. 2), whereas the second and third terms correspond to the vertex correlations (v.c., sometimes referred to as "exchange") matrix elements (diagrams (e) and $(\mathrm{d})$ ). We examine them separately, writing accordingly

$$
\left\langle p h^{-1}\left|j_{\mu}^{C}\right| F\right\rangle=\left\langle p h^{-1}\left|j_{\mu}^{C}\right| F\right\rangle_{\text {s.e. }}+\left\langle p h^{-1}\left|j_{\mu}^{C}\right| F\right\rangle_{\text {v.c. }} .
$$

The self-energy ph matrix element can be split into two parts, $\mathcal{H}_{p}^{\mu}$ and $\mathcal{H}_{h}^{\mu}$. The former corresponds to the diagram with the pion inserted in the particle line (Fig. $2 \mathrm{~g}$ ), whereas the latter describes the diagram with the pion inserted in the hole line (Fig. 2f). They are

$$
\begin{aligned}
\mathcal{H}_{p}^{\mu} & =-\frac{3 m f^{2}}{V^{2} m_{\pi}^{2} \sqrt{E_{\mathbf{p}} E_{\mathbf{h}}}} \sum_{\mathbf{k}} \frac{m}{E_{\mathbf{k}}} \bar{u}(\mathbf{p})(K-m) \frac{P-\not K}{(P-K)^{2}-m_{\pi}^{2}} S_{F}(P) \Gamma^{\mu}(Q) u(\mathbf{h}) \\
\mathcal{H}_{h}^{\mu} & =\frac{3 m f^{2}}{V^{2} m_{\pi}^{2} \sqrt{E_{\mathbf{p}} E_{\mathbf{h}}}} \sum_{\mathbf{k}} \frac{m}{E_{\mathbf{k}}} \bar{u}(\mathbf{p}) \Gamma^{\mu}(Q) S_{F}(H) \frac{(K-\not H)}{(K-H)^{2}-m_{\pi}^{2}}(K-m) u(\mathbf{h}),
\end{aligned}
$$

where the isospin trace has been performed.

Similarly, the vertex ph matrix element splits into two terms $\mathcal{F}^{\mu}$ and $\mathcal{B}^{\mu}$ representing the forward- and backward-going contributions, respectively (Fig. 2d and 2e). They are 


$$
\begin{aligned}
\mathcal{F}^{\mu} & =-\frac{m f^{2}}{V^{2} m_{\pi}^{2} \sqrt{E_{\mathbf{p}} E_{\mathbf{h}}}} \sum_{\mathbf{k}} \frac{m}{E_{\mathbf{k}}} \bar{u}(\mathbf{p}) \gamma_{5}(K-\not H) S_{F}(K+Q) \tau_{a} \Gamma^{\mu}(Q) \tau_{a} \gamma_{5} \frac{(K-m)}{(K-H)^{2}-m_{\pi}^{2}} u(\mathbf{h}) \\
\mathcal{B}^{\mu} & =-\frac{m f^{2}}{V^{2} m_{\pi}^{2} \sqrt{E_{\mathbf{p}} E_{\mathbf{h}}}} \sum_{\mathbf{k}} \frac{m}{E_{\mathbf{k}}} \bar{u}(\mathbf{p}) \frac{K-m}{(P-K)^{2}-m_{\pi}^{2}} \gamma_{5} \tau_{a} \Gamma^{\mu}(Q) \tau_{a} S_{F}(K-Q) \gamma_{5}(P-\not K) u(\mathbf{h}) .
\end{aligned}
$$

Finally, splitting also the electromagnetic nucleon operator $\Gamma^{\mu}$ into its isoscalar and isovector parts, one obtains the isoscalar and isovector contributions to the self-energy and vertex ph matrix elements. The final results can be cast in the form

$$
\begin{aligned}
\mathcal{H}_{p}^{\mu(S, V)} & =-\frac{3 f^{2}}{2 V^{2} m_{\pi}^{2} \sqrt{E_{\mathbf{p}} E_{\mathbf{h}}}} \\
& \times \sum_{\mathbf{k}} \frac{m}{E_{\mathbf{k}}} \bar{u}(\mathbf{p}) \frac{\not P-\not K}{(P-K)^{2}-m_{\pi}^{2}}(K-m)(P-\not K) S_{F}(P) \Gamma^{\mu(S, V)}(Q) u(\mathbf{h})
\end{aligned}
$$

and

$$
\begin{aligned}
\mathcal{H}_{h}^{\mu(S, V)} & =-\frac{3 f^{2}}{2 V^{2} m_{\pi}^{2} \sqrt{E_{\mathbf{p}} E_{\mathbf{h}}}} \\
& \times \sum_{\mathbf{k}} \frac{m}{E_{\mathbf{k}}} \bar{u}(\mathbf{p}) \Gamma^{\mu(S, V)}(Q) S_{F}(H)(K-\not H)(K-m) \frac{(K-\not H)}{(K-H)^{2}-m_{\pi}^{2}} u(\mathbf{h}),
\end{aligned}
$$

for the self-energy matrix elements and

$$
\begin{aligned}
\mathcal{F}^{\mu(S)} & =-\frac{3 m f^{2}}{V^{2} m_{\pi}^{2} \sqrt{E_{\mathbf{p}} E_{\mathbf{h}}}} \\
& \times \sum_{\mathbf{k}} \frac{m}{E_{\mathbf{k}}} \bar{u}(\mathbf{p}) \gamma_{5}(K-\not H) S_{F}(K+Q) \Gamma^{\mu(S)}(Q) \gamma_{5} \frac{(K-m)}{(K-H)^{2}-m_{\pi}^{2}} u(\mathbf{h}) \\
\mathcal{B}^{\mu(S)} & =-\frac{3 m f^{2}}{V^{2} m_{\pi}^{2} \sqrt{E_{\mathbf{p}} E_{\mathbf{h}}}} \\
& \times \sum_{\mathbf{k}} \frac{m}{E_{\mathbf{k}}} \bar{u}(\mathbf{p}) \frac{\not K-m}{(P-K)^{2}-m_{\pi}^{2}} \gamma_{5} \Gamma^{\mu(S)}(Q) S_{F}(K-Q) \gamma_{5}(P-\not K) u(\mathbf{h}),
\end{aligned}
$$

for the isoscalar and

$$
\mathcal{F}^{\mu(V)}=-\frac{m f^{2}}{V^{2} m_{\pi}^{2} \sqrt{E_{\mathbf{p}} E_{\mathbf{h}}}}
$$




$$
\begin{aligned}
& \times \sum_{\mathbf{k}} \frac{m}{E_{\mathbf{k}}} \bar{u}(\mathbf{p}) \gamma_{5}(K-\not H) S_{F}(K+Q) \Gamma^{\mu(V)}(Q)\left(\tau_{3}+i \varepsilon_{3 a b} \tau_{a} \tau_{b}\right) \gamma_{5} \frac{(K-m)}{(K-H)^{2}-m_{\pi}^{2}} u(\mathbf{h}) \\
& \mathcal{B}^{\mu(V)}=-\frac{m f^{2}}{V^{2} m_{\pi}^{2} \sqrt{E_{\mathbf{p}} E_{\mathbf{h}}}} \\
& \times \sum_{\mathbf{k}} \frac{m}{E_{\mathbf{k}}} \bar{u}(\mathbf{p}) \frac{\not K-m}{(P-K)^{2}-m_{\pi}^{2}} \gamma_{5} \Gamma^{\mu(V)}(Q)\left(\tau_{3}+i \varepsilon_{3 a b} \tau_{a} \tau_{b}\right) S_{F}(K-Q) \gamma_{5}(P-\not K) u(\mathbf{h}),
\end{aligned}
$$

for the isovector vertex ph matrix elements. Interestingly, as far as isospin traces are concerned, in the vertex matrix element the isoscalar/isovector ratio is -3, whereas in the selfenergy case it is 1 . Note that the MEC (pion-in-flight and seagull) ph matrix elements are purely isovector, whereas the vertex and self-energy correlations get both isoscalar and isovector contributions.

Note that the v.c. and s.e. ph matrix elements involve the nucleon propagator $S_{F}(P)$ which in some situations may imply the occurrence of singularities. In the case of the vertex diagrams, the four-momenta appearing in the propagators are $K+Q$ and $K-Q$ for the forward- (Fig. 2d) and backward-going (Fig. 2e) contributions, respectively, and an integration over $\mathbf{k}$ should be done. For $q \geq 2 k_{F}$ (no Pauli blocking) it can be proven (see appendix B) that only the forward diagram contains a pole, i.e., a value of the inner momentum $\mathbf{k}$ exists such that the nucleon carrying a four-momentum $K+Q$ is on-shell. In this situation the forward vertex ph matrix element is evaluated by taking the principal value in the integral over $\cos \theta_{k}$. In the case of the backward-going diagram the nucleon propagator $S_{F}(K-Q)$ has no singularity for the kinematics in which we are interested.

The case of the self-energy diagrams is different. Here the ph matrix element diverges, as the nucleons described by the Feynman propagators $S_{F}(P)$ and $S_{F}(H)$ are forced to be on-shell by energy and momentum conservation. Accordingly this term should in principle be renormalized. In [23] we present a detailed analysis of the different aspects of the impact of the self-energy term on the nuclear responses within the relativistic framework. A comparison with the non-relativistic reduction is also carried out there. Here we confine ourselves to computing the relativistic response functions through the hadronic tensor $W^{\mu \nu}$. As stated in Section 2, $W^{\mu \nu}$ can be evaluated using the current matrix elements or the polarization propagator. In the first instance, however, the presence of divergent ph matrix elements may lead to wrong responses or inconsistencies in the theory. In this paper, to avoid this happening, we calculate the s.e. contribution to the response with the polarization propagator.

An important point here is the fact that the hadronic tensor thus obtained, partly through the ph matrix elements and partly through the polarization propagator, is gauge invariant. This may be somewhat surprising because we show in appendix A that current conservation is already obtained at the level of the MEC and correlation ph matrix elements: hence the one-body current ph matrix element also has to be conserved. However, this occurs only in

\footnotetext{
${ }^{1}$ The latter result stems from the relation $\tau_{3}+i \varepsilon_{3 a b} \tau_{a} \tau_{b}=-\tau_{3}$; however we prefer to leave the isospin structure of the isovector exchange as in (33,34) since it makes more transaparent the self-energy-exchange cancellation in the continuity equation, as shown in appendix A.
} 
zeroth order of perturbation theory. To be dealt with properly, clearly the situation requires the renormalization of the ph energies and Dirac spinors. Only then does it become possible to set up a renormalized s.e. current which leads to a hadronic tensor that coincides with the one obtained here through the polarization propagator [23].

\section{Nuclear hadronic tensor and response functions}

In this Section we analyze the response functions for one-particle emission reactions within the RFG model. As discussed in the previous Section, the ph matrix elements corresponding to the different pionic diagrams are all well-defined except for the self-energy term which diverges. Therefore, on the one hand, in the case of the one-body, MEC and vertex correlation diagrams we evaluate the hadronic tensor starting from the current ph matrix elements (Fig. 2). On the other hand, for the self-energy diagrams we calculate the polarization propagator $\Pi^{\mu \nu}$.

The analysis of the nuclear hadronic tensor set up with the ph matrix elements has been presented in detail, within the RFG model, in previous papers [12, 20]. Hence, here we simply summarize the results needed for later discussions. The hadronic tensor that arises from the interference of the single-nucleon current, $j_{s . n}^{\mu}$. with the one-pion-exchange current $j_{a}^{\mu}$, with $a=S$ (seagull), $P$ (pion-in-flight) and v.c. (vertex), is for the RFG model with $Z=N$

$$
W^{\mu \nu}=\frac{3 Z}{8 \pi k_{F}^{3} q} \int_{h_{0}}^{k_{F}} h d h\left(\omega+E_{\mathbf{h}}\right) \int_{0}^{2 \pi} d \phi_{h} \sum_{s_{p}, s_{h}} 2 \operatorname{Re}\left[\left\langle p h^{-1}\left|\hat{j}_{s . n .}^{\mu}\right| F\right\rangle^{*}\left\langle p h^{-1}\left|\hat{j}_{a}^{\nu}\right| F\right\rangle\right]
$$

where $\left\langle p h^{-1}\left|\hat{j}_{s . n .}^{\mu}\right| F\right\rangle=\frac{m}{\sqrt{E_{\mathbf{p}}{ }_{\mathbf{h}}}} \bar{u}(\mathbf{p}) \Gamma^{\mu} u(\mathbf{h})$ is the single-nucleon ph matrix element with $\Gamma^{\mu}$ the electromagnetic nucleon current from eq. (10) and $\left\langle p h^{-1}\left|\hat{j}_{a}^{\nu}\right| F\right\rangle$ is the ph matrix element for the seagull, pion-in-flight or vertex current as given in eqs. (21), (22) and (27. 28), respectively.

Note that in eq. (35) the integral over hole polar angle, $\cos \theta_{h}$, has been performed explicitly by exploiting the energy conserving $\delta$-function. This fixes the minimum momentum of the hole according to

$$
h_{0}=m \sqrt{\varepsilon_{0}^{2}-1}, \quad \varepsilon_{0}=\operatorname{Max}\left\{\varepsilon_{F}-2 \lambda, \kappa \sqrt{1+\frac{1}{\tau}}-\lambda\right\}
$$

where the usual dimensionless variables

$$
\lambda=\frac{\omega}{2 m}, \quad \tau=\frac{\left|Q^{2}\right|}{4 m^{2}}, \quad \kappa=\frac{q}{2 m}, \quad \varepsilon_{F}=\frac{E_{F}}{m}, \quad \eta=\frac{h}{m}
$$

have been introduced and $E_{F}=\sqrt{k_{F}^{2}+m^{2}}$ is the Fermi energy. Moreover, the hole threemomentum

$$
\mathbf{h}=h\left(\sin \theta_{0} \cos \phi_{h}, \sin \theta_{0} \sin \phi_{h}, \cos \theta_{0}\right),
$$




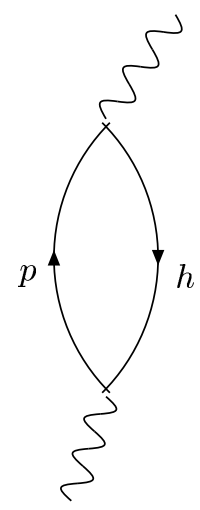

(a)

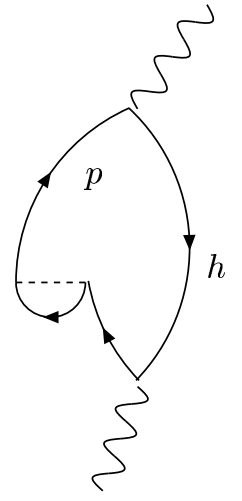

(b)

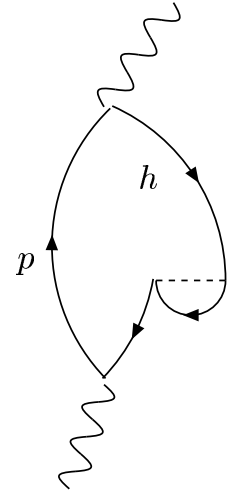

$(c)$

Figure 3: Feynman diagrams of the free (a) and first-order self-energy (b and c) polarization propagator.

involved in the hadronic tensor, must be evaluated for the following specific value of the polar angle

$$
\cos \theta_{0}=\frac{\lambda \varepsilon-\tau}{\eta \kappa}
$$

The hadronic tensor, as was the case for the current, can be also split into isoscalar and isovector parts, since there is no interference between the two isospin channels.

An important issue relates to the form factor of the $\pi N N$ vertex, $\Gamma_{\pi}$, which incorporates some aspects of the short-range physics affecting the pionic correlation. In all of the above expressions $\Gamma_{\pi}$ has not been explicitly indicated for sake of simplicity. Here we recall that its inclusion in the ph current matrix elements is not without consequences in connection with gauge invariance. In fact, in this case, the results quoted in appendix A are no longer valid unless new terms are added to the MEC (see [10, 20, 21]). Lacking a fundamental theory for $\Gamma_{\pi}$, in the calculations reported in the next Section we use the phenomenological expression

$$
\Gamma_{\pi}(P)=\frac{\Lambda^{2}-m_{\pi}^{2}}{\Lambda^{2}-P^{2}}
$$

with $\Lambda=1.3 \mathrm{GeV}$. As long as the dependence upon $\Lambda$ is not too strong the gauge invariance of the theory should not be too badly affected. Within a non-relativistic approach for the pion currents, a detailed discussion on the breakdown of the gauge invariance due to eq. (40), and on the dependence of the responses upon the cutoff value can be found in [20, 21].

Now, as already discussed, a crucial point to be emphasized is that the self-energy ph matrix element is divergent. Hence it should be renormalized to be used in the evaluation of the hadronic tensor. Referring the reader to [23] for a thorough discussion on this subject, here we show how the hadronic tensor is built by evaluating the polarization propagator corresponding to the two self-energy diagrams shown in Fig. 3. 
From the Feynman diagrams with the self-energy $\Sigma$ inserted in the particle or in the hole lines (Fig. 3), the polarization propagator is

$$
\begin{aligned}
\Pi^{\mu \nu}(Q)=-i \int \frac{d h_{0} d^{3} h}{(2 \pi)^{4}} & \operatorname{Tr}\left\{\Gamma^{\mu}(Q) S(H) \Sigma(H) S(H) \Gamma^{\nu}(-Q) S(H+Q)\right. \\
+ & \left.\Gamma^{\mu}(Q) S(H) \Gamma^{\nu}(-Q) S(H+Q) \Sigma(H+Q) S(H+Q)\right\}
\end{aligned}
$$

In the above the nucleon propagator within the RFG framework reads

$$
\begin{aligned}
S(K) & =\frac{K+m}{K-m+i \epsilon}+2 \pi i(K+m) \theta\left(k_{F}-k\right) \delta\left(K^{2}-m^{2}\right) \theta\left(k_{0}\right) \\
& =(K+m)\left[\frac{\theta\left(k-k_{F}\right)}{K^{2}-m^{2}+i \epsilon}+\frac{\theta\left(k_{F}-k\right)}{K^{2}-m^{2}-i \epsilon k_{0}}\right]
\end{aligned}
$$

Concerning the self-energy one has

$$
\Sigma(P)=-\frac{f^{2}}{V m_{\pi}^{2}} \sum_{\mathbf{k}, s_{k}, t_{k}} \frac{m}{E_{\mathbf{k}}} \tau_{a} \gamma_{5}(P-K) \frac{u(\mathbf{k}) \bar{u}(\mathbf{k})}{(P-K)^{2}-m_{\pi}^{2}} \tau_{a} \gamma_{5}(P-K) .
$$

In the following we illustrate the calculation by treating in detail the case of the selfenergy in the hole line. The analysis of the diagram with the s.e. insertion on the particle line is similar and then we shall just quote the final result. In appendix $\mathrm{C}$ we discuss the more general case with any number of self-energy insertions in the particle and/or in the hole lines.

Using the nucleon propagator in eq. (42), we can write the polarization propagator with the s.e. on the hole line as follows

$$
\begin{aligned}
\Pi^{\mu \nu}(Q)= & -i \operatorname{Tr} \int \frac{d h_{0} d^{3} h}{(2 \pi)^{4}} \Gamma^{\mu}(Q)(H+m) \Sigma(H)(\not H+m) \\
\times & {\left[\frac{\theta\left(h-k_{F}\right)}{\left(H^{2}-m^{2}+i \epsilon\right)^{2}}+\frac{\theta\left(k_{F}-h\right)}{\left(H^{2}-m^{2}-i \epsilon h_{0}\right)^{2}}\right] \Gamma^{\nu}(-Q)(\not H+\varnothing+m) } \\
& \times\left[\frac{\theta\left(|\mathbf{h}+\mathbf{q}|-k_{F}\right)}{(H+Q)^{2}-m^{2}+i \epsilon}+\frac{\theta\left(k_{F}-|\mathbf{h}+\mathbf{q}|\right)}{(H+Q)^{2}-m^{2}-i \epsilon\left(h_{0}+q_{0}\right)}\right] .
\end{aligned}
$$

Then, exploiting the identity 12]

$$
\frac{1}{\left(H^{2}-m^{2}-i \epsilon\right)^{2}}=\left.\frac{d}{d \alpha}\right|_{\alpha=0} \frac{1}{H^{2}-\alpha-m^{2}-i \epsilon},
$$

and subtracting the (infinite) vacuum polarization (see appendix C) we get

$$
\begin{aligned}
\Pi^{\mu \nu}(Q)= & -\left.i \frac{d}{d \alpha}\right|_{\alpha=0} \operatorname{Tr} \int \frac{d h_{0} d^{3} h}{(2 \pi)^{4}} \Gamma^{\mu}(Q)(\not H+m) \Sigma(H)(H+m) \Gamma^{\nu}(-Q)(\not H+\varnothing+m) \\
& \times\left[\frac{\theta\left(k_{F}-h\right)}{H^{2}-\alpha-m^{2}-i \epsilon h_{0}}\right]\left[\frac{\theta\left(|\mathbf{h}+\mathbf{q}|-k_{F}\right)}{(H+Q)^{2}-m^{2}+i \epsilon}\right] .
\end{aligned}
$$


Now the integral over the hole energy $h_{0}$ can be computed. For this purpose we rewrite the denominators of the hole and particle propagators in the form

$$
\begin{aligned}
H^{2}-\alpha-m^{2}-i \epsilon h_{0} & =H^{2}-m^{\prime 2}-i \epsilon h_{0}=\left(h_{0}+E_{\mathbf{h}}^{\prime}-i \epsilon\right)\left(h_{0}-E_{\mathbf{h}}^{\prime}-i \epsilon\right) \\
(H+Q)^{2}-m^{2}+i \epsilon & =\left(h_{0}+q_{0}+E_{\mathbf{p}}-i \epsilon\right)\left(h_{0}+q_{0}-E_{\mathbf{p}}+i \epsilon\right),
\end{aligned}
$$

where we have introduced a mass

$$
m^{\prime}=m^{\prime}(\alpha) \equiv \sqrt{\alpha+m^{2}}
$$

and an energy

$$
E_{\mathbf{h}}^{\prime}=E_{\mathbf{h}}^{\prime}(\alpha) \equiv \sqrt{\mathbf{h}^{2}+m^{\prime 2}}
$$

that depend upon the parameter $\alpha$ and we have used the relations $\mathbf{p}=\mathbf{h}+\mathbf{q}, E_{\mathbf{p}}=\sqrt{\mathbf{p}^{2}+m^{2}}$. Therefore the poles are located at the points

$$
\begin{aligned}
& h_{0}= \pm E_{\mathbf{h}}^{\prime}+i \epsilon \\
& h_{0}= \pm\left(E_{\mathbf{p}}-i \epsilon\right)-q_{0}
\end{aligned}
$$

and the integral over $h_{0}$ can be computed by closing the integration path in the lower halfplane, so that only the pole at $h_{0}=E_{\mathbf{p}}-i \epsilon-q_{0}$ contributes. Then the polarization propagator is found to be

$$
\begin{aligned}
\left.\stackrel{\Pi^{\mu \nu}(Q)}{=-\left.\frac{d}{d \alpha}\right|_{\alpha=0}} \operatorname{Tr} \int \frac{d^{3} h}{(2 \pi)^{3}} \Gamma^{\mu}(Q)(H+m) \Sigma(H)(\not H+m) \Gamma^{\nu}(-Q)(H+\varnothing+m)\right|_{h_{0}=E_{\mathbf{p}}-q_{0}} \\
\quad \times \frac{1}{2 E_{\mathbf{p}}} \frac{\theta\left(k_{F}-h\right) \theta\left(|\mathbf{h}+\mathbf{q}|-k_{F}\right)}{\left(E_{\mathbf{p}}-q_{0}\right)^{2}-\mathbf{h}^{2}-m^{\prime 2}-i \epsilon\left(E_{\mathbf{p}}-q_{0}\right)}
\end{aligned}
$$

The hadronic tensor then follows from formula (5) and reads

$$
\begin{aligned}
& W^{\mu \nu}=-\frac{1}{\pi} \operatorname{Im}\left[\Pi^{\mu \nu}(Q)\right] \\
& =\left.\left.\frac{3 \pi^{2} Z}{k_{F}^{3}} \frac{d}{d \alpha}\right|_{\alpha=0} \operatorname{Tr} \int \frac{d^{3} h}{(2 \pi)^{3}} \Gamma^{\mu}(Q)(H+m) \Sigma(H)(H+m) \Gamma^{\nu}(-Q)(H+\varnothing+m)\right|_{h_{0}=E \mathbf{p}-q_{0}} \\
& \times \frac{1}{2 E_{\mathbf{p}}} \theta\left(k_{F}-h\right) \theta\left(|\mathbf{h}+\mathbf{q}|-k_{F}\right) \frac{1}{2\left(E_{\mathbf{p}}-q_{0}\right)} \delta\left(E_{\mathbf{p}}-q_{0}-E_{\mathbf{h}}^{\prime}\right)
\end{aligned}
$$

where we have exploited the inequality $E_{\mathbf{p}}-q_{0}>0$ and the relation $\delta\left(x^{2}-x_{0}^{2}\right)=\delta\left(x-x_{0}\right) / 2 x_{0}$ for $x_{0}>0$. 
Finally, using the energy-conserving delta-function and setting $p_{0}=E_{\mathbf{p}}$, we obtain

$$
\begin{aligned}
W^{\mu \nu}=\left.\frac{3 \pi^{2} Z}{k_{F}^{3}} \frac{d}{d \alpha}\right|_{\alpha=0} & \left.\operatorname{Tr} \int \frac{d^{3} h}{(2 \pi)^{3}} \Gamma^{\mu}(Q)(H+m) \Sigma(H)(H+m) \Gamma^{\nu}(-Q)(\not P+m)\right|_{h_{0}=E_{\mathbf{h}}^{\prime}(\alpha)} \\
& \times \frac{1}{2 E_{\mathbf{p}}} \theta\left(k_{F}-h\right) \theta\left(|\mathbf{h}+\mathbf{q}|-k_{F}\right) \frac{1}{2 E_{\mathbf{h}}^{\prime}(\alpha)} \delta\left(E_{\mathbf{p}}-q_{0}-E_{\mathbf{h}}^{\prime}(\alpha)\right)
\end{aligned}
$$

an expression that coincides with the one given by eq. (93) in appendix C.

Using a similar analysis, the hadronic tensor with the self-energy inserted in the particle line turns out to read (see appendix C)

$$
\begin{aligned}
W^{\mu \nu}=\left.\frac{3 \pi^{2} Z}{k_{F}^{3}} \frac{d}{d \beta}\right|_{\beta=0} & \left.\operatorname{Tr} \int \frac{d^{3} h}{(2 \pi)^{3}} \Gamma^{\mu}(Q)(H+m) \Gamma^{\nu}(-Q)(P+m) \Sigma(P)(\not P+m)\right|_{p_{0}=E_{\mathbf{p}}^{\prime}(\beta)} \\
& \times \frac{1}{2 E_{\mathbf{p}}^{\prime}(\beta)} \theta\left(k_{F}-h\right) \theta\left(|\mathbf{h}+\mathbf{q}|-k_{F}\right) \frac{1}{2 E_{\mathbf{h}}} \delta\left(E_{\mathbf{p}}^{\prime}(\beta)-q_{0}-E_{\mathbf{h}}\right)
\end{aligned}
$$

with $E_{\mathbf{p}}^{\prime}(\beta)=\sqrt{\mathbf{p}^{2}+m^{\prime 2}}$ and $m^{\prime}=m^{\prime}(\beta)=\sqrt{\beta+m^{2}}$.

In the above expressions, after the derivatives with respect to the parameters $\alpha$ and $\beta$ are taken, the integral over the hole polar angle $\cos \theta_{h}$ can be performed analytically by exploiting the $\delta$-function. Hence the s.e. contribution to the hadronic tensor can finally be expressed as a double integral. Since the self-energy $\Sigma$ involves a triple integral, the contribution to hadronic tensor turns out to be a 5-dimensional integral, to be carried out numerically.

\section{Results}

In this Section we report the numerical results obtained for the pionic MEC (pion-in-flight and seagull) and for the correlation (vertex and self-energy) contributions to the quasielastic peak in the $1 \mathrm{p}-1 \mathrm{~h}$ sector. The calculation is fully relativistic. We have taken $Z=N=20$ and set $k_{F}=237 \mathrm{MeV} / \mathrm{c}$, which is representative of nuclei in the vicinity of ${ }^{40} \mathrm{Ca}$.

The 5-dimensional integrations of the MEC and correlation responses implicit in eq. (35) have been performed numerically. The reliability of the numerical procedure has been proven by checking that the free RFG responses coincide with their analytical expressions (see, e.g., [26]). Moreover the transverse MEC responses have been compared with the nonrelativistic calculation developed in [15], where the seagull ph matrix element is evaluated analytically, while the pion-in-flight contribution is reduced to a one-dimensional integral: both calculations give the same results for $q$ and $k_{F}$ small.

\subsection{MEC}

With regard to the MEC we have found the following:

- They are almost irrelevant in the longitudinal channel, whereas they are small but not insignificant in the transverse one. This outcome emerges from inspection of Figs. 4 and 

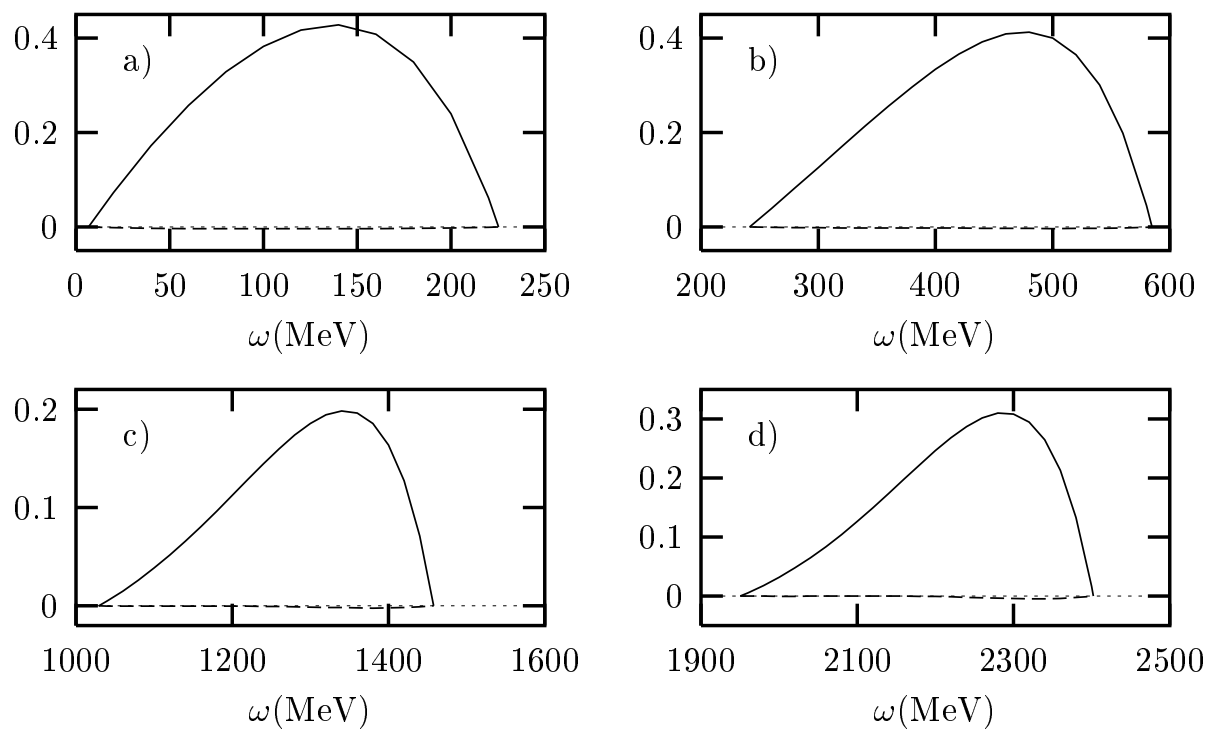

Figure 4: Longitudinal response versus $\omega$. Solid: free; dashed: MEC contribution. Here and in all of the figures to follow the nucleus is ${ }^{40} \mathrm{Ca}$ with $k_{F}=237 \mathrm{MeV} / \mathrm{c}$ and the units are $10^{-1}$ $\mathrm{MeV}^{-1}$ at $q=0.5 \mathrm{GeV} / \mathrm{c}$ (panel a), $10^{-2} \mathrm{MeV}^{-1}$ at $q=1 \mathrm{GeV} / \mathrm{c}$ (panel b), $10^{-3} \mathrm{MeV}^{-1}$ at $q=2 \mathrm{GeV} / \mathrm{c}$ (panel c) and $10^{-4} \mathrm{MeV}^{-1}$ at $q=3 \mathrm{GeV} / \mathrm{c}$ (panel d).
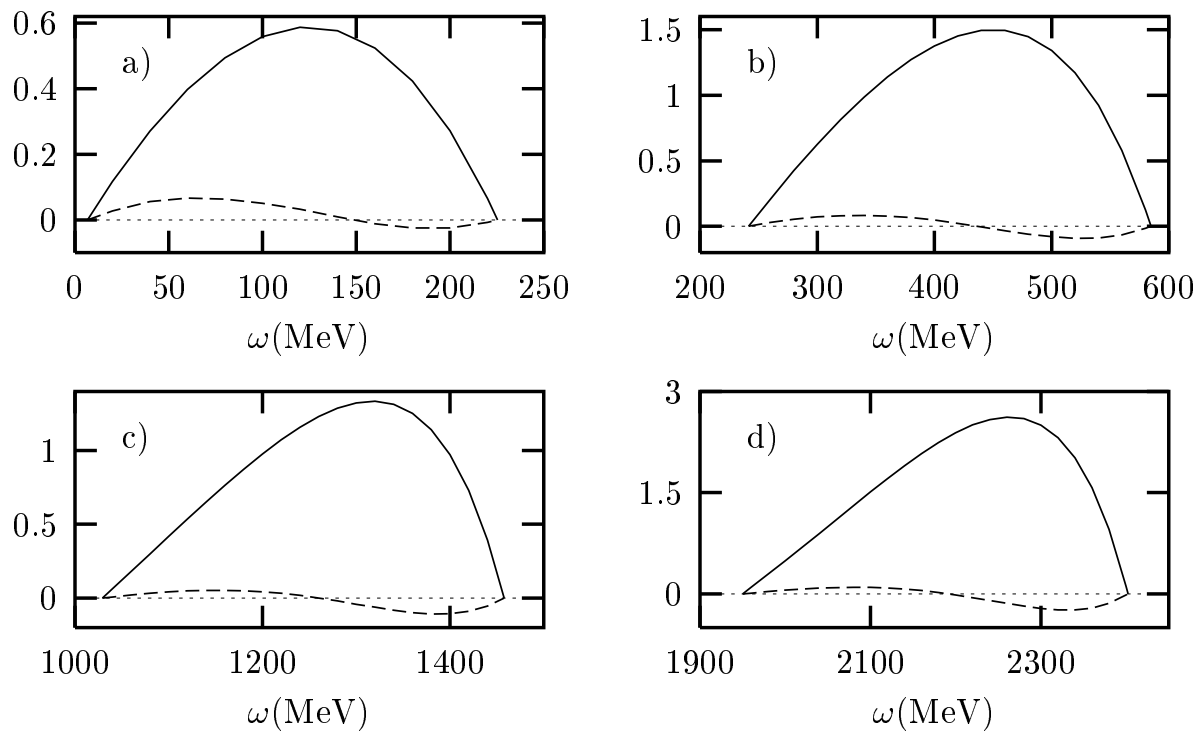

Figure 5: Transverse response versus $\omega$ at $q=0.5$ (a), 1 (b), 2 (c) and 3 (d) GeV/c. Solid: free; dashed: MEC contribution. 
5, where the MEC contribution (dashed curves) to the $\mathrm{L}$ and $\mathrm{T}$ responses is displayed for various values of $q$ together with the free responses (solid). While in the $\mathrm{L}$ channel the MEC are hardly visible, in the T channel they contribute somewhat more, although the contributions there typically only amount to about $5-10 \%$, depending upon $q$ and $\omega$ (see later). This dominance of the spin interaction between the photon and the MEC prompts us to concentrate on the transverse channel.
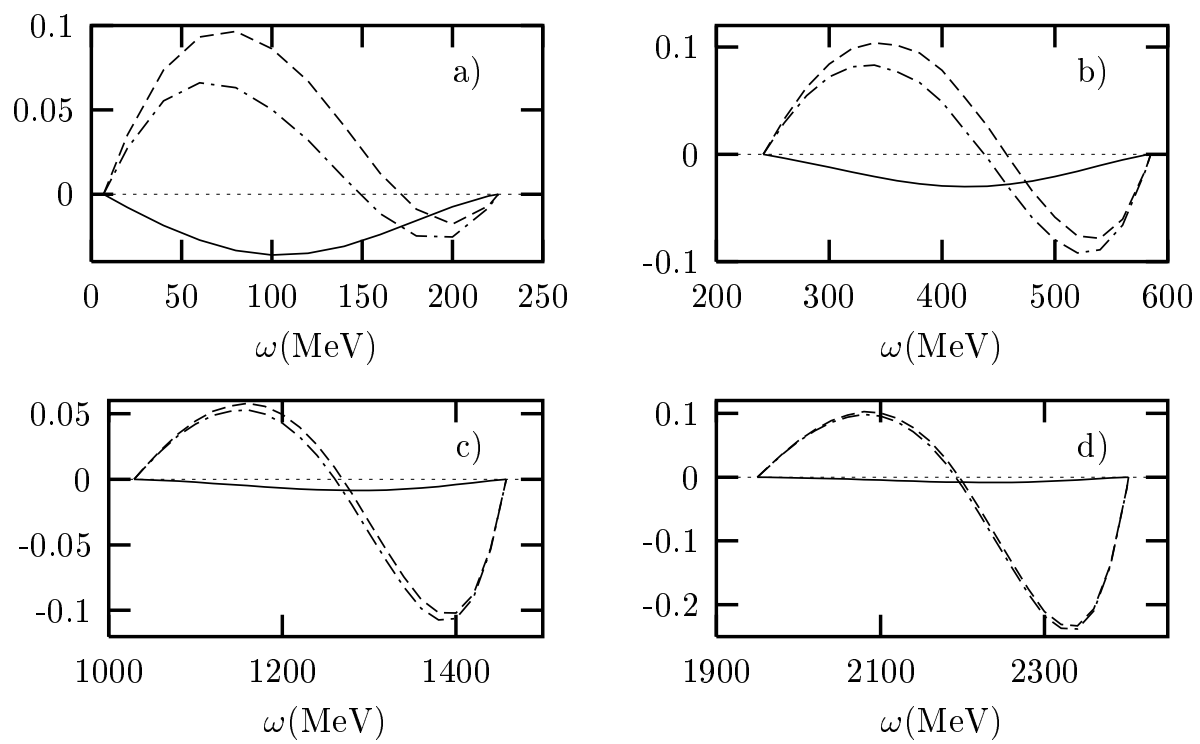

Figure 6: MEC contribution to transverse response versus $\omega$ at $q=0.5$ (a), 1 (b), 2 (c) and 3 (d) $\mathrm{GeV} /$ c. Solid: pion-in-flight; dashed: seagull; dot-dashed: total MEC.
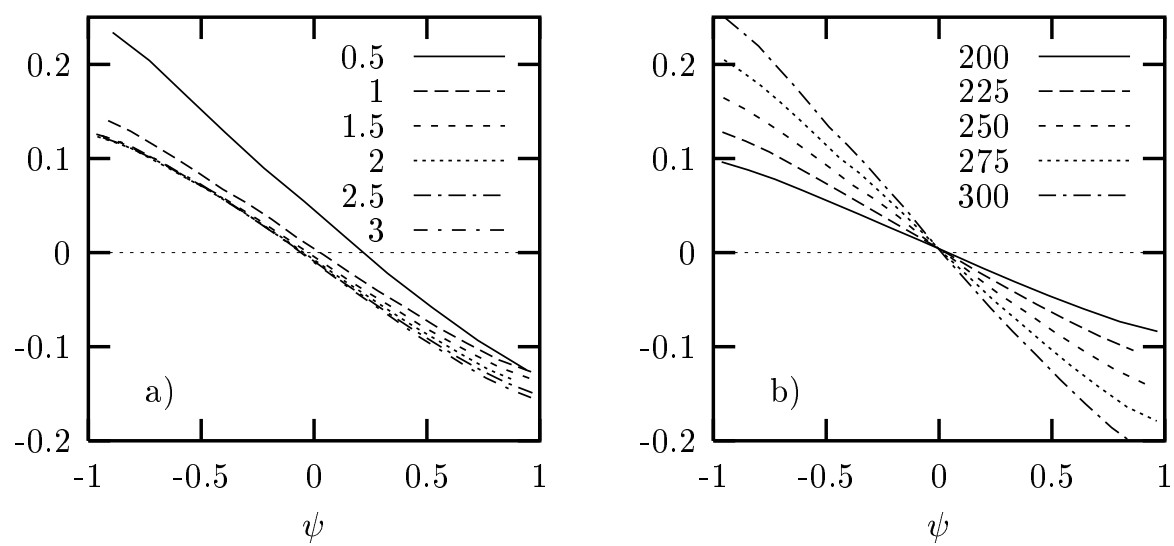

Figure 7: The ratio MEC/free in the transverse channel plotted versus $\psi$ at $k_{F}=237 \mathrm{MeV} / \mathrm{c}$ for various values of $q\left(\right.$ in $\mathrm{GeV} / \mathrm{c}$ ) in panel a and for various values of $k_{F}($ in $\mathrm{MeV} / \mathrm{c}$ ) at $q=1$ $\mathrm{GeV} / \mathrm{c}$ in panel b. 
- In Fig. 6 we display the separate pion-in-flight and seagull contribution to $R^{T}$ for various values of $q$. It appears that the seagull term (dashed lines) is always larger than the pion-in-flight term (solid lines), this dominance increasing with $q$ and again reflecting the spin nature of the photon-MEC interaction. Moreover whereas the pionin-flight term is always negative, the seagull changes sign with $\omega$, inducing a (mild) softening of the response.

- In Fig. 7a we study the evolution with $q$ of the MEC: it clearly appears that their relative contribution to $R^{T}$ decreases with $q$, but does not vanish for large values of q. This point is best illustrated by displaying the response versus the scaling variable 24

$$
\psi \equiv \frac{\varepsilon_{0}-1}{\varepsilon_{F}-1},
$$

using eq. (36). Indeed the range $-1 \leq \psi \leq 1$ will then be common to all responses, no matter what is the value of $q$. In the figure we plot the ratio $\rho^{T}=R_{M E C}^{T} / R_{\text {free }}^{T}$ for $q=0.5,1,1.5,2,2.5$ and $3 \mathrm{GeV} / \mathrm{c}$ : we see the relative MEC contribution decrease in going from 0.5 to $1 \mathrm{GeV} / \mathrm{c}$, but then it rapidly saturates at or slightly above $q=1$ $\mathrm{GeV} / \mathrm{c}$, where its value stabilizes, typically around $10 \%$. Thus, one can see from these results that at momentum transfers above $1 \mathrm{GeV} / \mathrm{c}$ scaling of the first kind is satisfied for the MEC contributions considered in this work. Note also that, for high $q$ the MEC almost vanish in the vicinity of the QEP $(\psi=0)$.

- In Fig. 7b we investigate the $k_{F}$ dependence of the MEC contribution. Importantly, the latter is seen to grow with $\boldsymbol{k}_{F}$, in contrast with the free response which decreases as $k_{F}^{-1}$. This point is again best studied by displaying the response as a function of $\psi$, since the response region broadens with $k_{F}$. In Fig. $7 \mathrm{~b}$ the ratio $\rho^{T}$ is plotted versus $\psi$ at $q=1 \mathrm{GeV} / \mathrm{c}$ for $k_{F}$ varying from 200 to $300 \mathrm{MeV} / \mathrm{c}$. It clearly appears that the relative MEC contribution grows with $k_{F}$ (attaining a value of about $20 \%$ at $k_{F}=300$ $\mathrm{MeV} / \mathrm{c})$.

Indeed, our earlier studies of the current matrix elements [19] can be used in analyzing the functional dependence on $k_{F}$. There a "semi-relativistic" approach was followed in which, once the leading power dependence on the dimensionless variable $h / m$ was identified, where $h=|\mathbf{h}|$ is the three-momentum of any particle below the Fermi surface, all higher-order terms in $h / m$ are neglected, since $h / m$ is bounded by $\eta_{F}=$ $k_{F} / m$ and the latter is generally quite small. For the matrix elements this was shown in 19 to be an excellent approximation. Here we see a detailed verification of those ideas. In fact, a full analysis at very small Fermi momentum shows that the seagull contribution has a leading power of $k_{F}^{2}$, while the pion-in-flight contribution begins as $k_{F}^{4}$ - the former because it involves one- and two-body spin currents, the latter because it involves convection currents and therefore one higher power of momentum in both the one- and two-body contributions. Thus, if we divide the fully-relativistic results obtained in this work by these respective factors of $k_{F}^{n}$ with $n=2,4$ for seagull and pion-in-flight, respectively, we should see very little residual dependence on $k_{F}$, at least at very low Fermi momentum. Indeed, even for values of the Fermi momentum 
that are typical of nuclei across the periodic table this is still roughly the case, as can be seen by examining Fig. 8, panels $a$ and $b$. The relatively small remaining spread with varying $k_{F}$ reflects the quality of the semi-relativistic treatment in which only the leading $k_{F}$ dependence is taken into account. Of course, in the present work no approximation is made at all and it is not necessary to use the rough power dependence except as an orientation to assess the degree to which the two-body MEC effects depend on the density in a manner that is different from the behaviour seen in the one-body currents.

It is also interesting to note at this juncture that, if we attempt to find the best choice for the power $n$ in the two cases, with $n$ free to be non-integer, specifically for nuclei in the range $200<k_{F}<260$ (i.e., excluding only the lightest nuclei), then the respective powers turn out to be somewhat lower than 2 and 4 , namely, about 1.5 and 2.5.

Given that once the leading powers of $k_{F}$ are removed the integrals remaining in the two cases are of roughly similar magnitude, one might expect the pion-in-flight contribution to be much smaller than the seagull contribution, at least at high $q$ where such arguments can be made relatively easily (at low $q$ the pion propagators, etc. make the argument less secure). Indeed, having powers of $k_{F}^{4}$ and $k_{F}^{2}$, as discussed above, would imply that at high $q$ typically the seagull will win by roughly a factor of $\left(m / k_{F}\right)^{2} \cong 16$, and this is borne out in Fig. 6 .

Thus, overall the seagull contribution is dominant and so an overall leading- $k_{F}$ dependence of about $k_{F}^{2}$ is appropriate. Since the RFG has a factor of $k_{F}^{-1}$ this means that the relative dependence - these two-body $1 \mathrm{p}-1 \mathrm{~h}$ pionic contributions compared with the RFG - goes approximately as $k_{F}^{3}$. That is, when studying the scaling behavior of the second kind, these two-body MEC processes violate the second-kind scaling by roughly three powers of $\boldsymbol{k}_{F}$. Clearly the effect is a rapid function of the Fermi momentum (or equivalently, of the density): for example, if one considers the cases ${ }^{2} \mathrm{H} /{ }^{4} \mathrm{He} /$ heavy nuclei with Fermi momenta of approximately 55/200/260 MeV/c, respectively, then the $1 \mathrm{p}-1 \mathrm{~h}$ MEC contributions amount to $0.1 / 5 / 10 \%$ of the total transverse response, respectively (normalizing to $10 \%$ for the heavy nucleus case - see the first bullet).

- In Fig. 9 we compare the MEC contribution to the transverse response with the nonrelativistic calculation of [15] for $q=500 \mathrm{MeV} / \mathrm{c}$. For this comparison we use $\Gamma_{\pi}=1$ and the static pion propagator in the relativistic calculation. The effect of static versus dynamic pion propagator will be discussed in the next item. From Fig. 9 we see that, apart from the difference stemming from the relativistic kinematics which shrinks the response domain, the relativistic responses are about $30 \%$ smaller than the non-relativistic ones, indicating that relativity plays an important role even for not so high $q$-values.

- Finally the impact on the responses of the relativistic propagator $\Delta_{\pi}(K)=\left(K^{2}-\right.$ $\left.m_{\pi}^{2}\right)^{-1}$ as compared to the static one $\Delta_{\pi}^{(n . r .)}(\mathbf{k})=-\left(\mathbf{k}^{2}+m_{\pi}^{2}\right)^{-1}$, which is commonly used in non-relativistic calculations, is explored. In Fig. 10 the pion-in-flight, seagull and total MEC contributions to $R^{T}$ are evaluated for $q=0.5$ and $2 \mathrm{GeV} / \mathrm{c}$ using both 

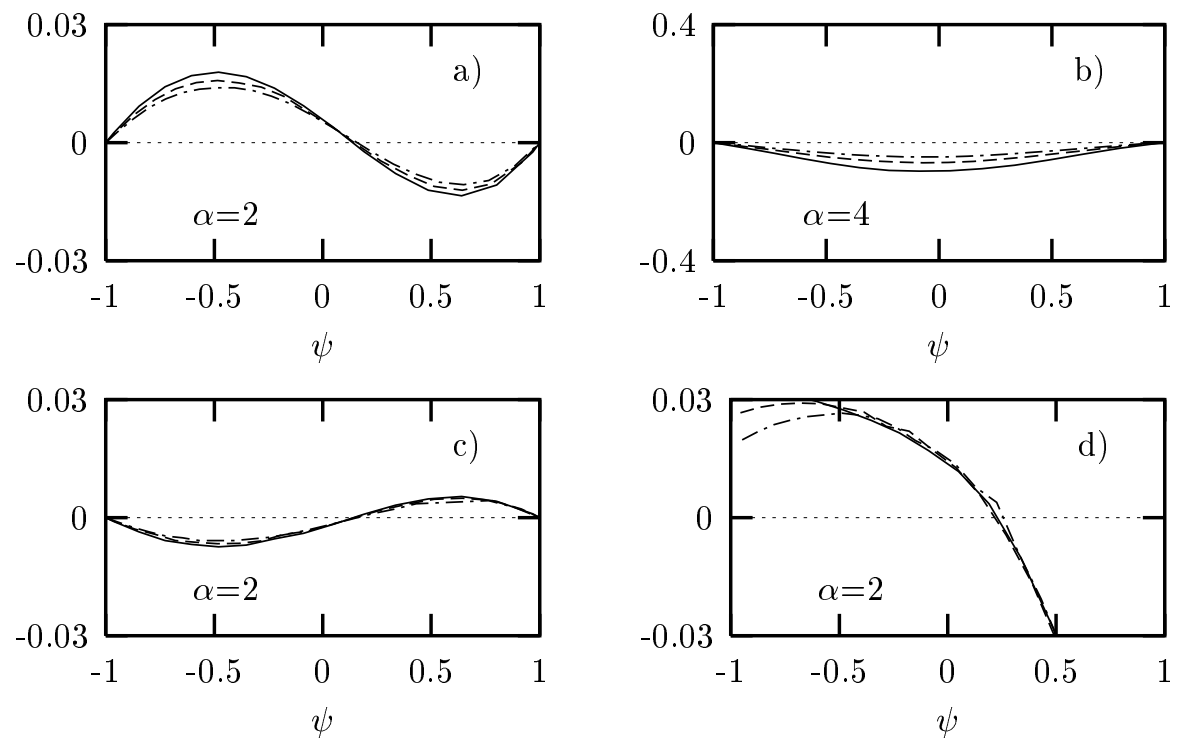

Figure 8: The ratio $R^{T} / k_{F}^{\alpha}$ of the seagull (a), pion-in-flight (b), vertex correlations (c) and self-energy (d) contributions to the transverse response and $k_{F}^{\alpha}$ (with $\alpha$ as indicated in each panel) is plotted versus $\psi$ for $k_{F}=200$ (solid), 250 (dashed) and 300 (dot-dashed) MeV/c. The momentum transfer is $q=1 \mathrm{GeV} / \mathrm{c}$.

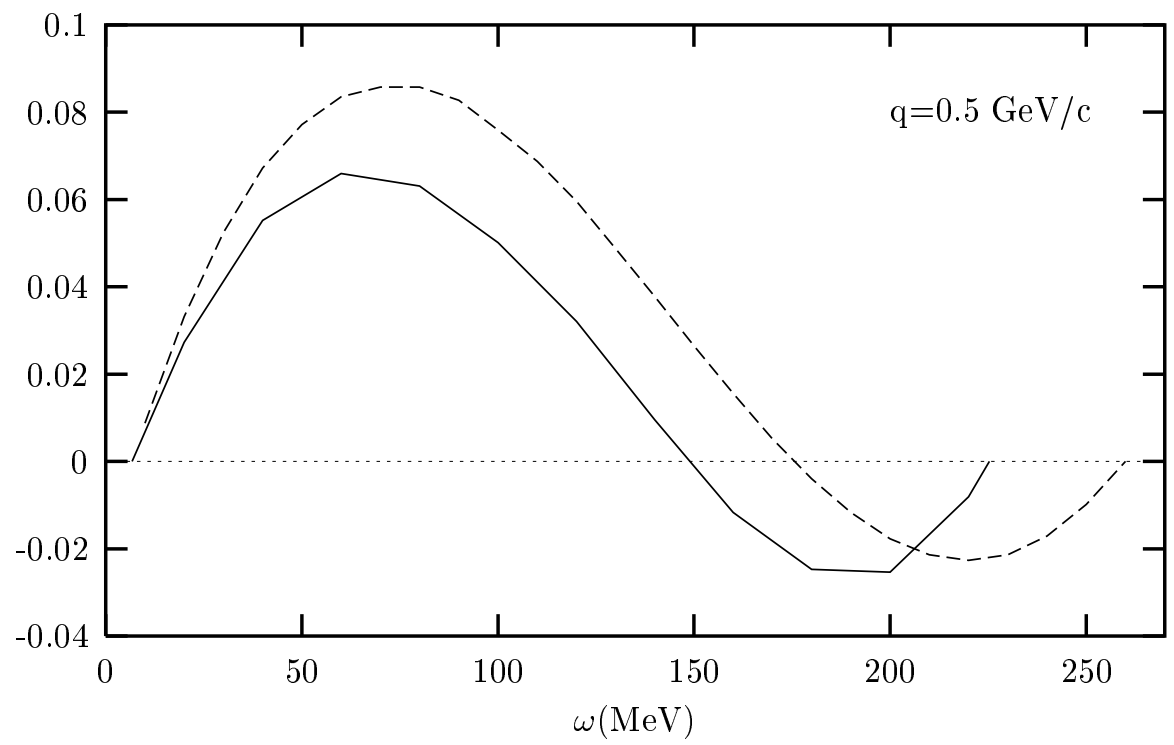

Figure 9: Transverse MEC contribution (solid) compared with the non-relativistic calculation of [15] (dashed). 
propagators: it appears that the dynamical propagator affects the pion-in-flight more than the seagull term (it increases the latter by more than a factor 2 at $q=2 \mathrm{GeV} / \mathrm{c}$ ); however, the two effects tend to cancel, so that their net effect is not very significant.

\section{$5.2 \quad$ Vertex correlations}

Where the v.c. are concerned we have found the following:

- Their action, while substantial in both the longitudinal and transverse channel, is actually dominant in the former by roughly a factor of 3:1, as is evident from Figs. 11 and 12 , where the v.c. contribution (dashed) displayed for several values of $q$ together with the free responses (solid). This outcome relates to the minor role played by the isoscalar contribution in the transverse response, in turn due to the smallness of the isoscalar magnetic moment. Worth pointing out is the oscillatory behavior versus $\omega$ of the vertex correlations, which induces a hardening of the responses.

In addition the seagull and vertex correlations tend to cancel in the transverse channel, especially for low values of $q$, whereas for higher $q$ the MEC dominate (see Fig. 13). Note that both the seagull and v.c. exactly vanish at the same value of $\omega$, the latter coinciding with the QEP for high momentum transfers.

- In Fig. 14 we study the evolution with $q$ of the v.c. in the longitudinal and transverse channel respectively plotting the ratios $\rho^{L(T)}=R_{v . c .}^{L(T)} / R_{\text {free }}^{L(T)}$ at $q=0.5,1,1.5,2,2.5$ and $3 \mathrm{GeV} / \mathrm{c}$. Clearly the v.c. do not saturate quite as rapidly as the MEC, although their behaviour is rather similar and saturation again occurs somewhere above $q=1$ $1.5 \mathrm{GeV} / \mathrm{c}$ : thus again scaling of the first kind is achieved at high momentum transfers for these contributions. Moreover, similarly to the MEC case, for high $q$ the v.c. almost vanish around the $\mathrm{QEP}(\psi=0)$. In fact the v.c. contributions and the seagull MEC contributions are rather similar in shape, but opposite in sign (cf. Figs. 7a and 14 (right panel)). Thus, they tend to cancel and, since the relative magnitude of the seagull to the v.c. is roughly $2: 1$, the net effect of the two together is to behave the way the former does but with roughly $1 / 2$ its strength.

- In Fig. 15 we explore the $k_{F}$ dependence of the vertex correlations. They are found to grow with $k_{F}$, much as the MEC do. Again from a semi-relativistic point of view, expanding only in powers of hole three-momenta over nucleon mass, we find a leading behaviour for $k_{F}$ small that goes as $k_{F}^{2}$. In Fig. 15 the ratios $\rho^{L}$ and $\rho^{T}$ are plotted versus $\psi$ at $q=1 \mathrm{GeV} / \mathrm{c}$ for $k_{F}$ varying from 200 to $300 \mathrm{MeV} / \mathrm{c}$ and we clearly see that the v.c. contribution grows with $k_{F}$. As in the MEC seagull case, for Fermi momenta in the typical range (200-250 MeV/c) a slightly lower power of $k_{F}^{3 / 2}$ actually provides the best fit to the full results. The range seen in Fig. 8c reflects the (weak) dependence on $k_{F}$ of terms ignored in the semi-relativistic approximation. The basic conclusion is similar to that made above for the seagull contribution and hence the total MEC at high $q$, namely, scaling of the second kind is badly broken by effects that go roughly as $k_{F}^{3}$. 

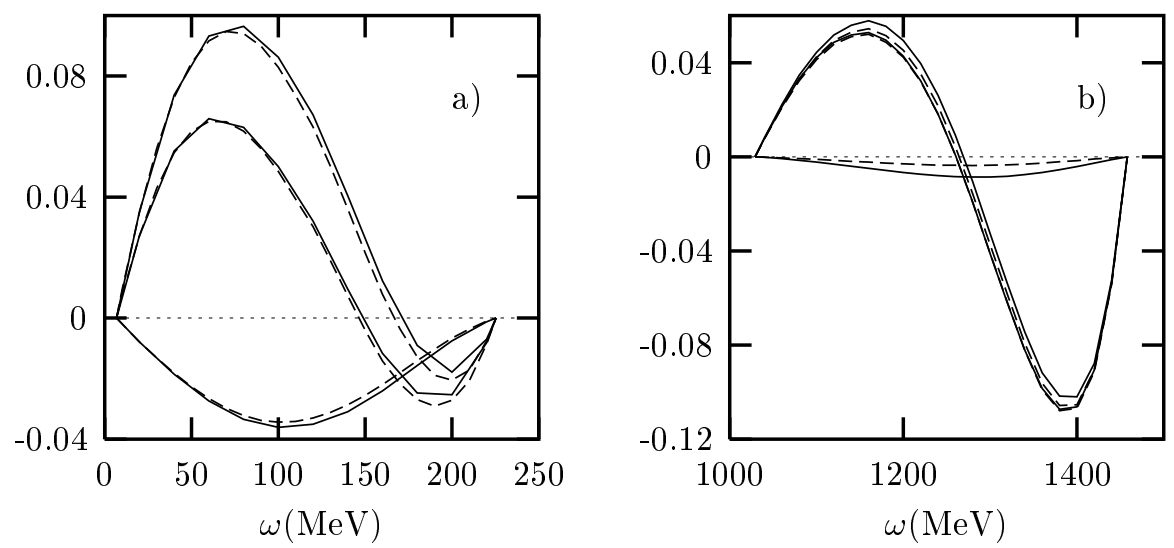

Figure 10: MEC contribution to $R^{T}$ versus $\omega$ with dynamic (solid curves) and static (dashed curves) pion propagator at $q=0.5$ (a) and 2 (b) GeV/c. The separate pion-in-flight and seagull contributions are displayed.
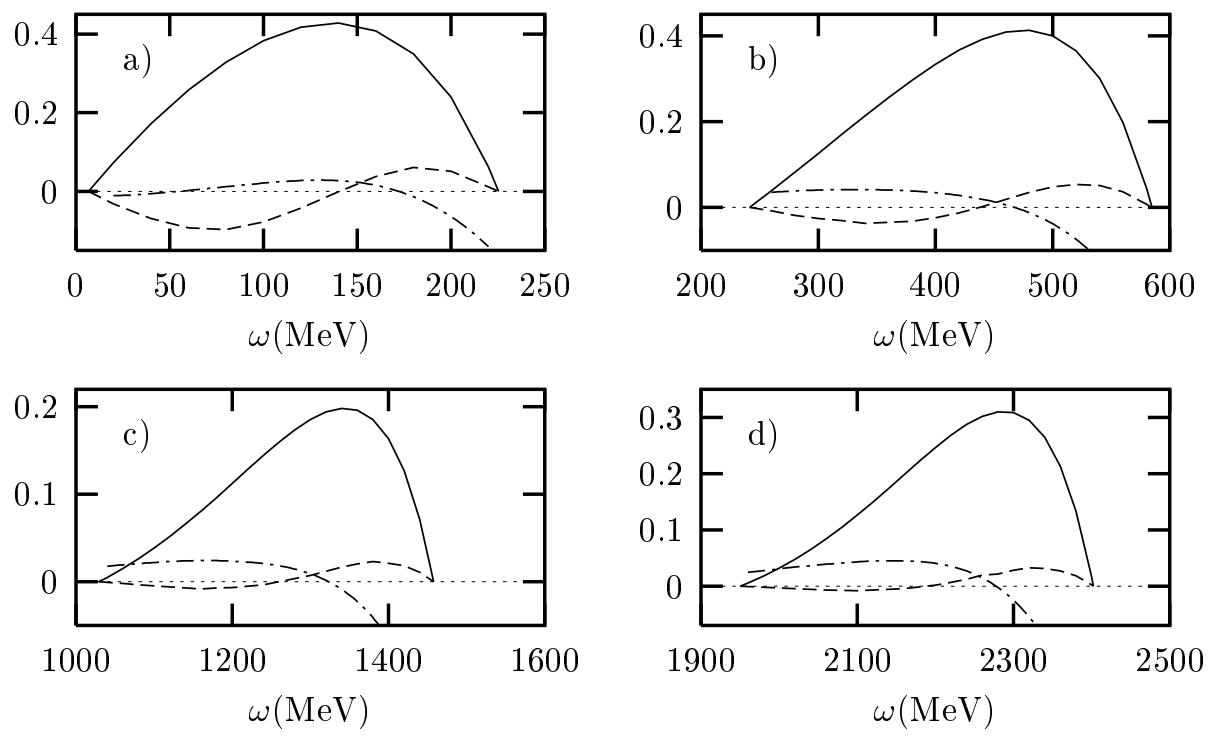

Figure 11: Correlation current contributions to $R^{L}$ versus $\omega$ at $q=0.5$ (a), 1 (b), 2 (c) and 3 (d) $\mathrm{GeV} / \mathrm{c}$. Solid: free; dot-dashed: self-energy; dashed: vertex correlations. 

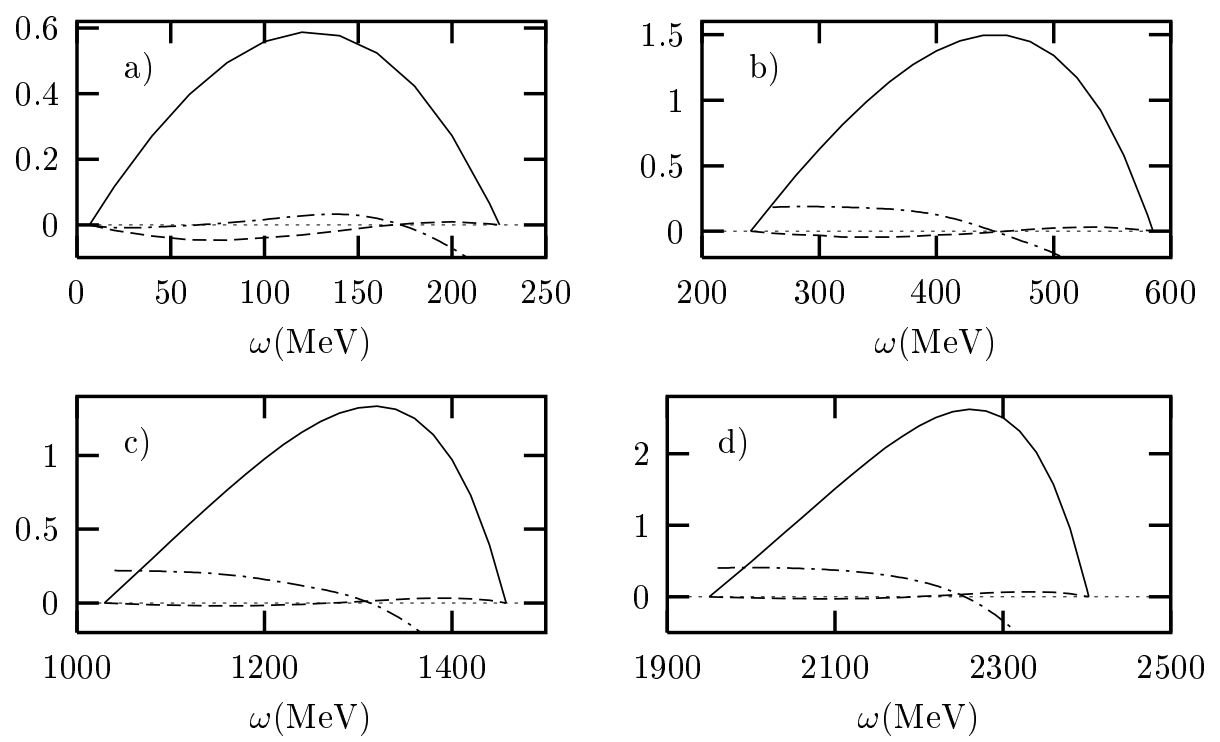

Figure 12: Correlation current contributions to $R^{T}$ versus $\omega$ at $q=0.5$ (a), 1 (b), 2 (c) and 3 (d) $\mathrm{GeV} / \mathrm{c}$. Solid: free; dot-dashed: self-energy; dashed: vertex correlations.
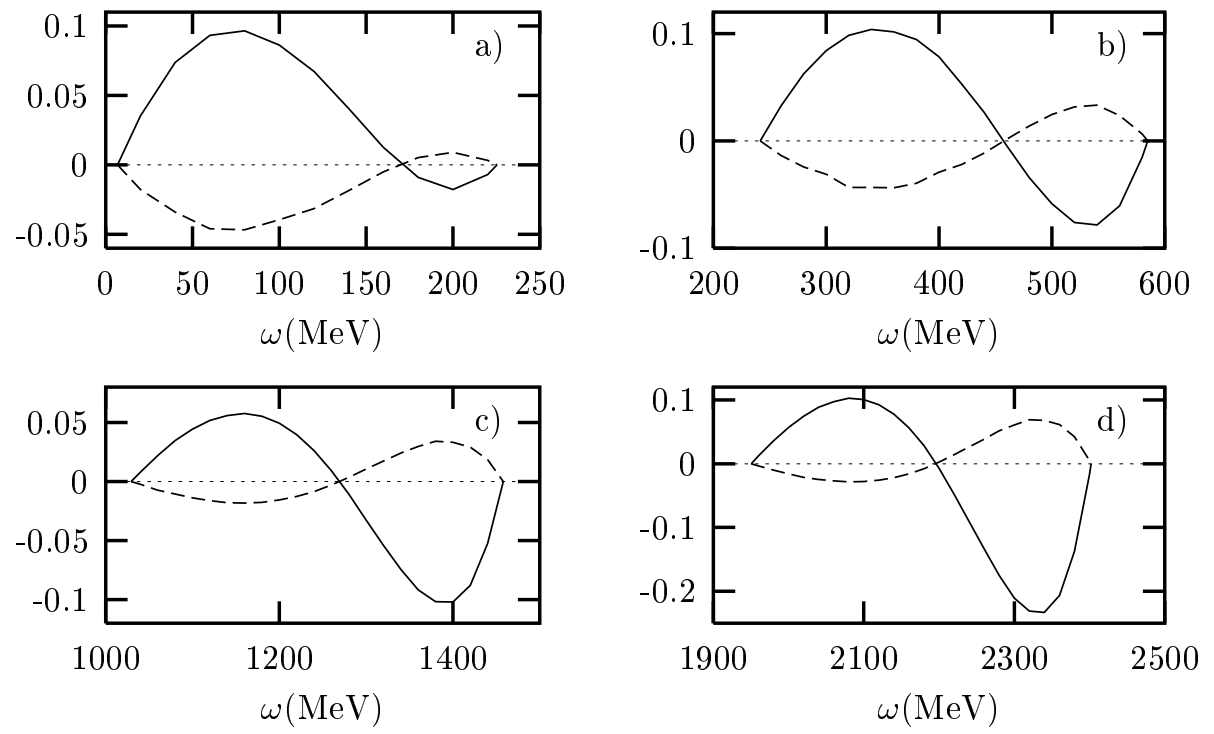

Figure 13: Seagull (solid) and v.c. (dashed) contributions to $R^{T}$ plotted versus $\omega$ at $q=0.5$ (a), 1 (b), 2 (c) and 3 (d) GeV/c. 

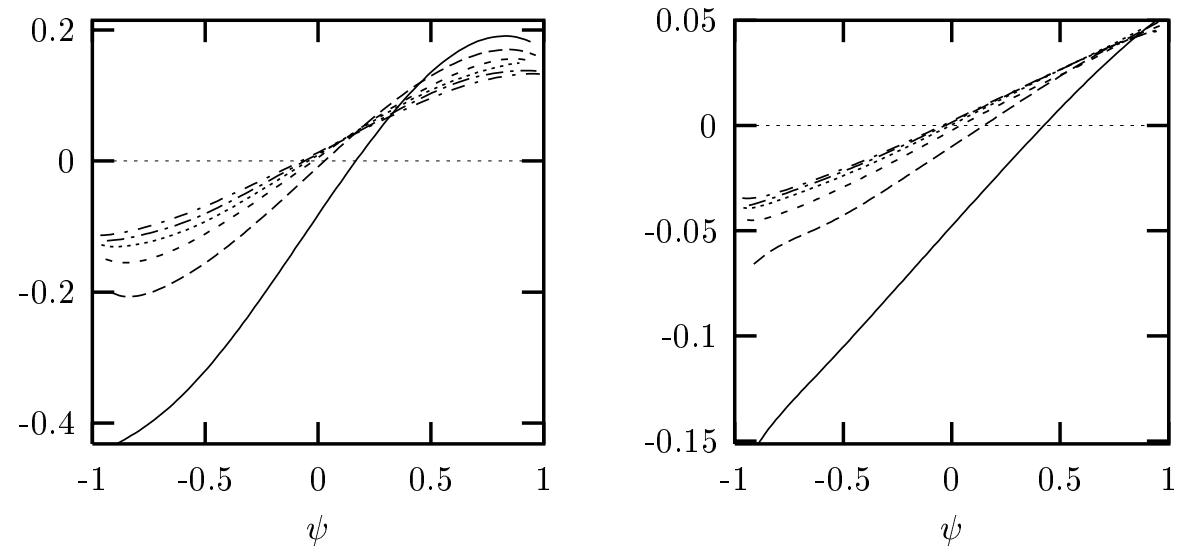

Figure 14: The ratio (v.c.)/free in the longitudinal (left panel) and transverse (right panel) channel plotted versus $\psi$ at $k_{F}=237 \mathrm{MeV} / \mathrm{c}$ for various $q$. The curves are labelled as in Fig. 7a.
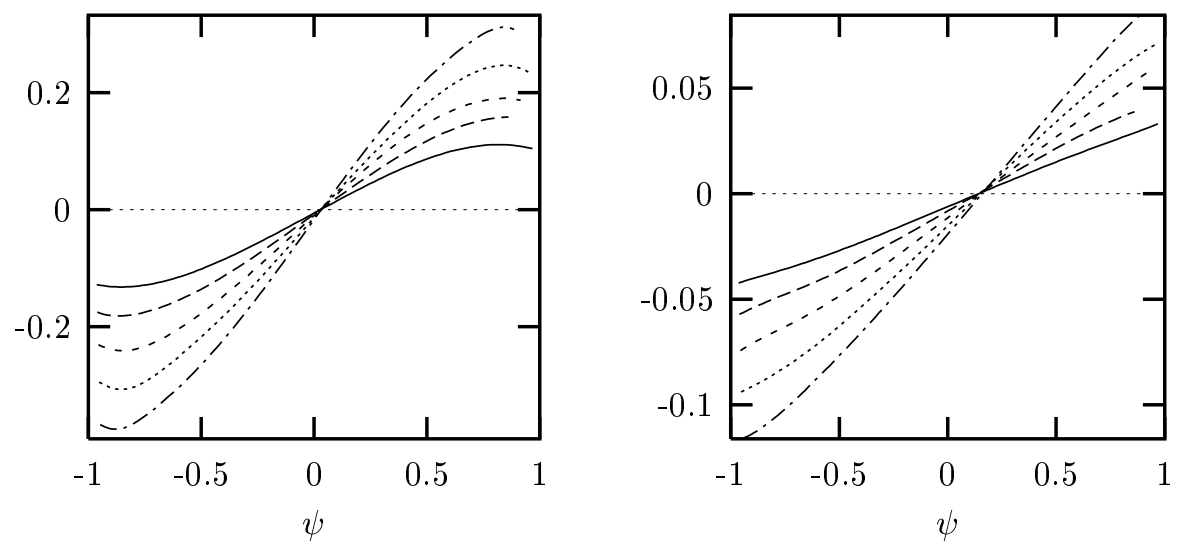

Figure 15: The ratio (v.c.)/free in the longitudinal (left panel) and transverse (right panel) channel plotted versus $\psi$ at $q=1 \mathrm{GeV} / \mathrm{c}$ for various $k_{F}$. The curves are labelled as in Fig. $7 \mathrm{~b}$. 


\subsection{Self energy}

We have found that the self-energy contribution results from a quite delicate cancellation between the responses having only the particle or only the hole dressed, as shown in Fig. 16 in the longitudinal channel for $q=0.5,1,2$ and $3 \mathrm{GeV} / \mathrm{c}$ (similar results hold in the transverse case). This was already pointed out in [20] within the framework of a treatment in which relativistic effects were partially incorporated, and it is now confirmed here in a fully relativistic context.

Whereas this cancellation is very substantial at $q=0.5 \mathrm{GeV} / \mathrm{c}$, as the momentum transfer increases the imbalance between the two contributions grows. Indeed the response associated with the particle self-energy is suppressed by the form factors and by the pion propagator, but that coming from the hole self-energy is not. As a result, for $q \geq 2 \mathrm{GeV} / \mathrm{c}$ the total selfenergy response almost coincides with the hole result alone and induces a moderate softening to the free response. Note that the s.e. contribution does not vanish on the borders of the response region. Moreover for high values of $\omega$ (close to the upper border) it becomes very large (see Figs. 11 and 12) and yields a vanishing (or even negative) response, clearly pointing to the insufficiency of a first-order perturbative treatment in this kinematical region. This effect was already present in the partially relativized analysis of 20 and, significantly, it is emphasized by our fully relativistic calculation. Therefore the summation of the full Fock series becomes necessary in this case.

Finally we explore the scaling and superscaling properties of the self-energy correlations by studying their momentum transfer- and density-dependence. In Fig. 17 we plot the ratio $\rho^{L}=R_{\text {s.e. }}^{L} / R_{\text {free }}^{L}$ versus $\psi$ for the particle (panel a), hole (panel b) and total (panel c) selfenergies at $q=0.5,1,2$ and $3 \mathrm{GeV} / \mathrm{c}$. As expected, the particle contribution decreases with $q$, going to zero at $q \simeq 2 \mathrm{GeV} / \mathrm{c}$, whereas the hole contribution, although also decreasing with $q$ when not too high, saturates for $q \geq 1 \mathrm{GeV} / \mathrm{c}$. As a result the total self-energy, displayed in panel (c), grows with $q$ (in contrast to all other cases considered above) in the range $q=0.5-2 \mathrm{GeV} / \mathrm{c}$, then stabilizes typically at about $30-40 \%$ of the free response to the left of the QEP, thus inducing the above-mentioned softening of the longitudinal response. Similar results are found in the transverse channel. In summary, again scaling of the first kind is achieved at momentum transfers somewhat below $2 \mathrm{GeV} / \mathrm{c}$.

In Fig. 18 we display the same ratio for three different values of the Fermi momentum at $q=1 \mathrm{GeV} / \mathrm{c}$. It appears that, as for the other two-body correlations, the self-energy relative contribution grows with $k_{F}$, specifically $R_{\text {s.e. }}^{L} \propto k_{F}^{2}$, although not uniformly in $\psi$ (see Fig. 8, panel $d$ ) - recall that in the first-order analysis presented in this paper the edges of the response region are not treated adequately for the self-energy contribution and thus should not be taken too seriously. Where the self-energy contribution is correctly modeled (away from the edges) we again see breaking of second-kind scaling by roughly $k_{F}^{3}$.

To complete the presentation of our results we display in Figs. 19 and 20 the global responses in first order of perturbation theory and compare them with the zeroth order ones (free responses) for several momentum transfers. Here one assesses the impact of the global two-body current contribution to the responses. First the overall effect of the two- 

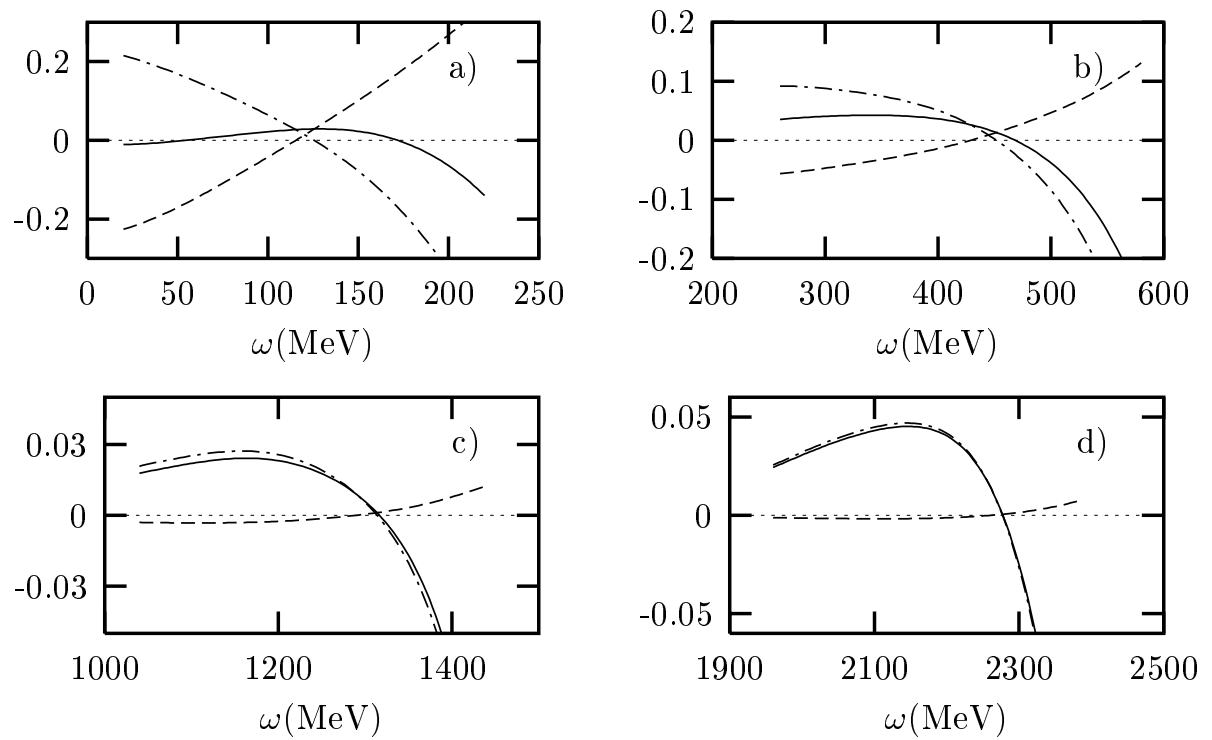

Figure 16: Particle (dashed) and hole (dot-dashed) contributions to the longitudinal self-energy (solid) versus $\omega$ for $q=0.5$ (a), 1 (b), 1.5 (c) and 2 (d) GeV/c.
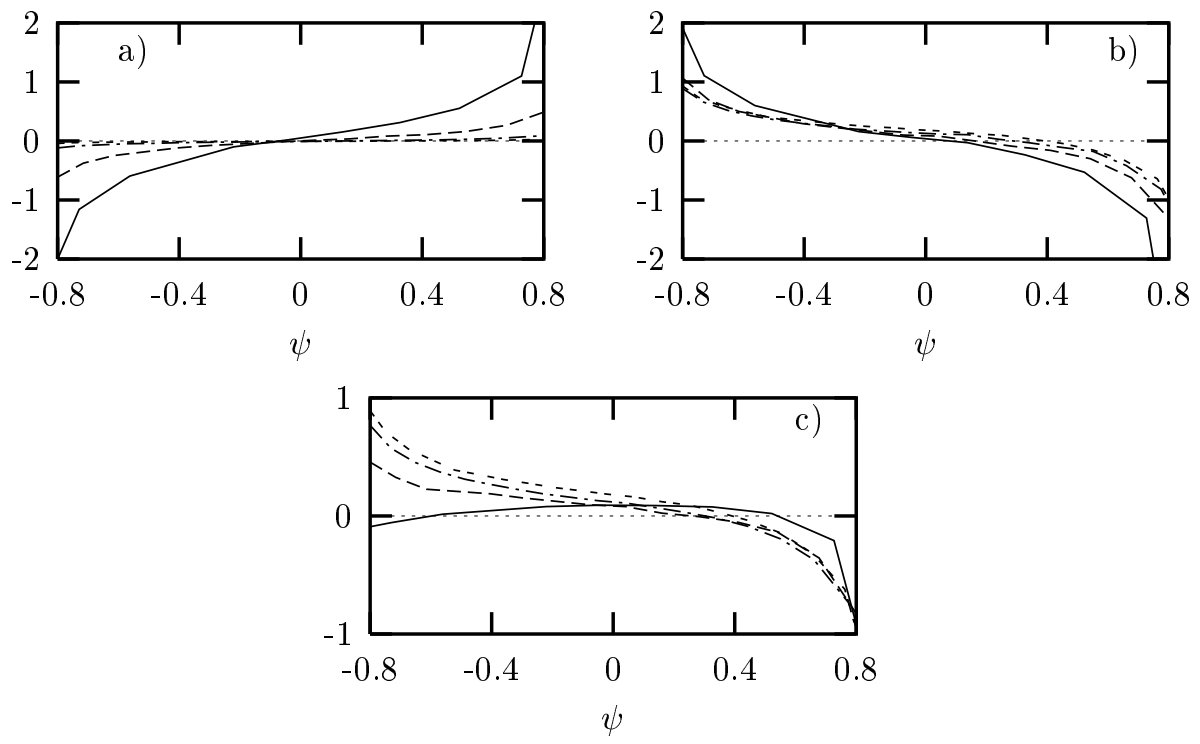

Figure 17: The ratio of the particle (a), hole (b) and total (c) s.e. contribution to the free longitudinal response is plotted versus $\psi$ for $q=0.5$ (solid), 1 (dashed), 2 (dot-dashed) and 3 (short-dashed) $\mathrm{GeV} / \mathrm{c}$. 

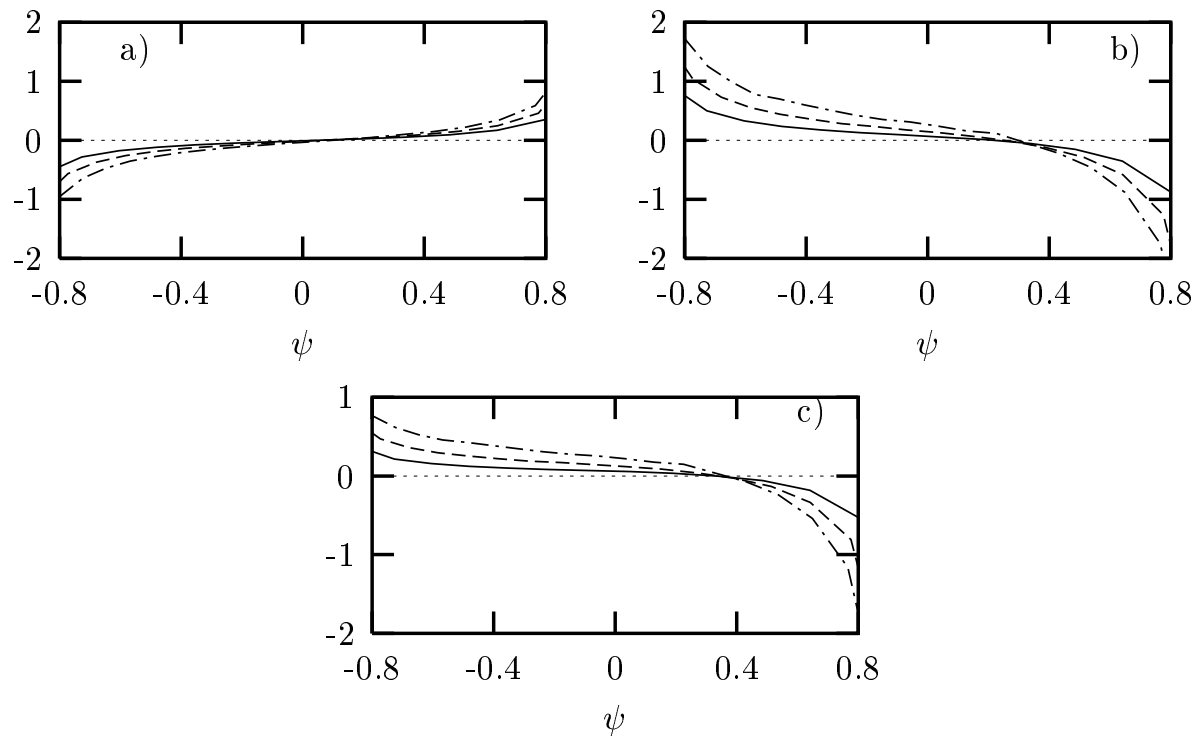

Figure 18: The ratio of the particle (a), hole (b) and total (c) s.e. contribution to the free longitudinal response is plotted versus $\psi$ for $k_{F}=200$ (solid), 250 (dashed) and 300 (dot-dashed) $\mathrm{MeV} / \mathrm{c}$ and $q=1 \mathrm{GeV} / \mathrm{c}$.
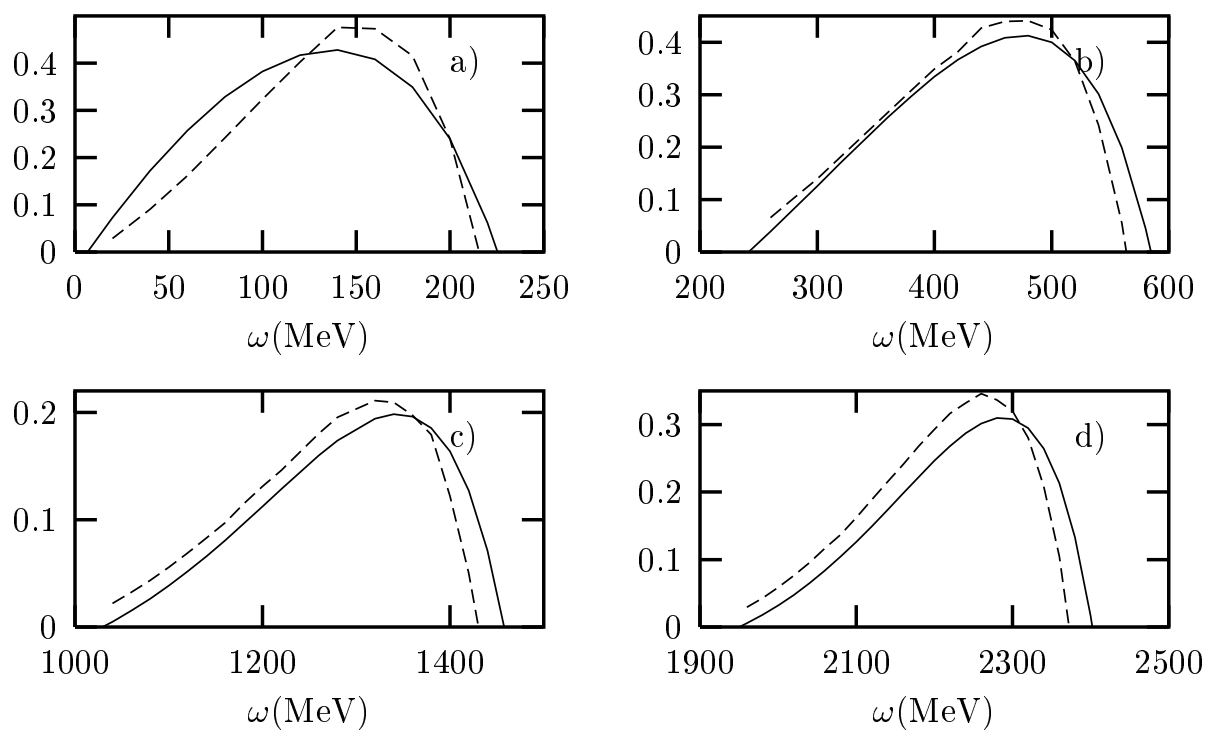

Figure 19: Longitudinal response versus $\omega$ including all first-order contributions (dashed) compared with the free result (solid) at $q=0.5$ (a), 1 (b), 2 (c) and 3 (d) GeV/c. 

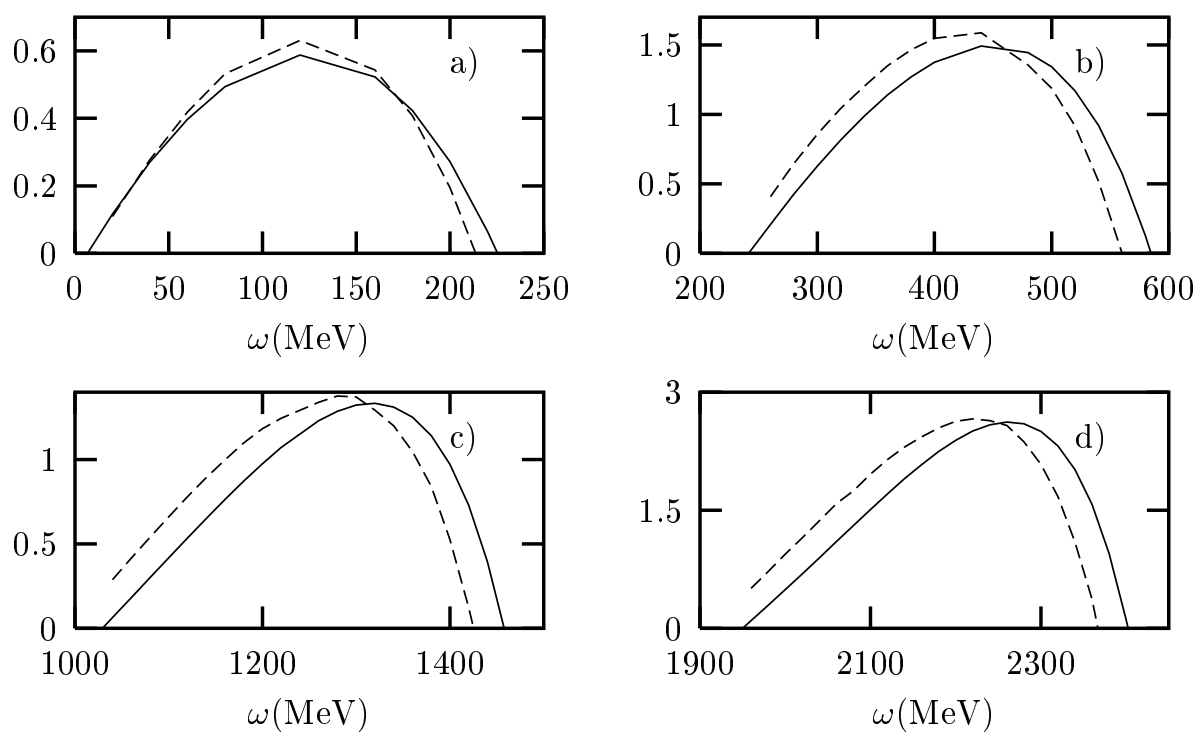

Figure 20: Transverse response versus $\omega$ including all first-order contributions (dashed) compared with the free result (solid) at $q=0.5$ (a), 1 (b), 2 (c) and 3 (d) GeV/c.

body currents appears sufficiently modest to justify our first-order treatment. Next the softening at large $q$ appears to be common to both L and T channels, whereas at low $q$ the longitudinal response displays a hardening that is absent in the transverse one. Also evident is the already-noted almost vanishing of the two-body correlation contribution at the peak of the free responses. Finally the unrealistic dominance of the self-energy contribution on the upper border is apparent.

\section{Conclusions}

In this paper we have addressed some of the ingredients that enter when attempting to model the longitudinal and transverse nuclear response functions for inclusive quasielastic electron scattering. Because of its complexity this problem cannot presently be viewed as solved: indeed, although many papers claim success in accounting for the data, a closer scrutiny reveals a different situation. It is not only that contributions left out in various analyses are far from being small, but, even more serious, fundamental physics principles (Lorentz covariance, gauge invariance and unitarity) turn out patently to be violated. Thus the successes in reproducing the experiments, regrettably few both in connection with the $\mathrm{L} / \mathrm{T}$ separation and with the set of nuclei or range of $q$ explored, often reflect more an adjusting of parameters than a real understanding of the physics involved in the QEP.

Searching for an improvement of this situation we have engaged in a more systematic approach to the problem, assuming as a zeroth-order approximation the RFG model. This appears justified on two counts: i) because RFG is Lorentz covariant, ii) because the surface of the nucleus is largely irrelevant in the QEP.

One of our basic themes has been the implementation of Lorentz covariance. In past 
work [19] we attempted to approximate the full theory by identifying a dimensionless variable that is small enough to be suitable to use in setting up a non-relativistic expansion of the responses (namely the momentum of a nucleon lying below the Fermi surface compared with its mass). In contrast, in the present work no expansion whatsoever is involved and our calculation fully respects relativity. The motivation for this should be clear: the theoretical analyses of the present and future experiments at TJNAF demand as full an implementation of relativity as is feasible.

Since the physics of the QEP is remote not only from the physical surface of the nucleus, but from the Fermi surface as well, we consider a treatment in terms of nucleonic and mesonic degrees of freedom (the latter viewed both as force and current carriers) to be appropriate. Hence, as a first step, we focus on pions as they can be expected under these conditions to be an important, if not the major, carrier of the currents that respond to an external electromagnetic field impinging on the nucleus. The pions, in our framework, are dealt with in first order of perturbation theory, since their effects on the free responses of the RFG are not expected to be too disruptive.

A point worth making in connection with all of the two-body currents considered in the present work is the following: their contributions appear to be quite small (if not vanishing) at the peak of the quasielastic responses. This corresponds to viewing the Fermi sphere to be split into two domains, an inner one associated with a sphere with a radius smaller than $k_{F}$, and the other corresponding to the spherical shell representing the difference between the two concentric spheres. The contributions tend to cancel one another and at the peak, where the whole Fermi sphere is involved in the response, they nearly do so completely. Of course the radius of the inner sphere and the sign of the contributions are current dependent.

Gauge invariance is a fundamental property we have also addressed in this work. In particular we have verified whether or not the continuity equation it implies is valid order by order in perturbation theory. Notably we have succeeded in showing that the continuity equation for the one-body (single-nucleon) and the two-body (MEC and correlations) currents is indeed fulfilled; hence our approach deals consistently with forces and currents. At the same time we have also shown how crucial relativity is in achieving this and how much care is needed in dealing with the ph matrix element of the self-energy current. Actually the present approach points to the necessity of renormalizing both the energies and the spinors in the self-energy ph matrix elements. Work under these lines is under progress [23.

As far as the self-energy contribution is concerned, the present results show that for high momentum transfers a first-order perturbative treatment seems to be insufficient to deal with the kinematical region corresponding to high values of $\omega$ (close to the upper border of the response region). This shortcoming demands the inclusion of higher-order perturbative terms, namely the summation of the whole Fock series, a task performed in a partially relativized framework [20] but not yet achieved in a fully relativistic one. It might be worth pointing out that the above shortcoming relates to the "wrong" analytic properties of our first-order polarization propagator: it should be a meromorphic function of the energy, yet it displays a double pole.

With regard to the other contributions of the two-body currents to $R^{L}$ and $R^{T}$, our results are highly satisfactory. Indeed for the MEC we have found that their contribution is small enough to be well handled in first order. In particular both the pion-in-flight and 
seagull contributions are very small in the $\mathrm{L}$ channel where the virtual photon exchanged between the electron and the Fermi gas couples to the charge of the pion. We thus see that the MEC affect the Coulomb sum rule very marginally. In the T channel instead the MEC are more significant and the following features emerge (see also Section 5 for itemized observations):

- the seagull contribution dominates, in accord with the spin nature of the MEC physics, and displays an oscillating behaviour versus $\omega$ yielding a (modest) softening of the response;

- the MEC contribution does not vanish when $q$ increases, but ultimately at very high $q$ it displays scaling of the first kind;

- the MEC contributions break the scaling of the second kind; i.e., they go as higher powers of $k_{F}$ than does the RFG. In fact, the total MEC contribution goes roughly as $k_{F}^{3}$ with respect to the RFG and thus when $k_{F}$ becomes small the MEC contribution tends to vanish. Hence, in going from the lightest nuclei where $k_{F}$ is as small as about $55 \mathrm{MeV} / \mathrm{c}$ (viz., for the deuteron) to heavy nuclei where it may be as large as $260 \mathrm{MeV} / \mathrm{c}$, one should see the 1p-1h quasielastic MEC contribution go from being essentially negligible to becoming typically $5-10 \%$ of the total transverse response.

With regard to the correlation contribution arising from the vertex corrections (v.c.), we have found that the $\mathrm{L}$ channel dominates over the $\mathrm{T}$ channel by a margin of roughly $3: 1$. The longitudinal response effectively picks up only these correlation contributions, since the MEC effects are so small there, and the former contribute to the total at roughly the 10$15 \%$ level. Indeed, were these to be the only contributions needed in addition to the RFG response itself, then we would expect the total to shift in $\omega$ (or in $\psi$ ). Since the correlation contributions are roughly symmetrical about the QEP, it is important to note that their impact on the Coulomb sum rule should be very small, perhaps only at the few percent level.

The correlation contribution to $R^{T}$ is similar to the MEC contribution, but is smaller, roughly $1 / 2$ the size of the latter. Since the two are of opposite sign they tend to cancel and thus the total is similar to the MEC contribution but is cut down by a factor of two. This statement is of course only approximate, since the correlation contribution attains its asymptotic value as a function of $q$ somewhat less rapidly than does the MEC contribution. Interestingly, both the seagull and v.c. contributions appear to vanish at exactly the same value of $\omega$. Overall the total (the sum of $1 \mathrm{p}-1 \mathrm{~h} \mathrm{MEC}+1 \mathrm{p}-1 \mathrm{~h}$ correlations) yields contributions to be added to the RFG that (1) do not go away as $q$ becomes very large, (2) attain scaling of the first kind at momentum transfers somewhat above $1 \mathrm{GeV} / \mathrm{c}$, while (3) they clearly violate scaling of the second kind, going approximately as $k_{F}^{3}$ relative to the RFG result (which of course scales).

In conclusion, in this paper we have pursued the program of a systematic analysis of the inclusive nuclear responses in the domain of the QEP and beyond. The motivation for this effort lies in the recognition that in this energy domain, unlike for the ground and low-lying excited states of nuclei, the interaction among the constituents of the system cannot be 
subsumed into a potential. Currents are important; and moreover the consistent treatment

of currents and forces is important. For the first time one is able to investigate the scaling behavior of at least some of the contributions to the inclusive cross section under high-energy conditions using a non-trivial (interacting) model in which issues relating to covariance and gauge invariance are addressed consistently.

\section{Acknowledgements}

This work was partially supported by funds provided by DGICYT (Spain) under Contracts Nos. PB/98-1111, PB/98-0676 and PB/98-1367 and the Junta de Andalucía (Spain), by the Spanish-Italian Research Agreement HI1998-0241, by the "Bruno Rossi" INFN-CTP Agreement, by the INFN-CICYT exchange and in part by the U.S. Department of Energy under Cooperative Research Agreement No. DE-FC02-94ER40818.

\section{Appendix A. The gauge invariance of the two-body currents ph matrix elements}

Following the study presented in Section 2 of gauge invariance at the level of the freespace particle-particle matrix elements, here we extend the analysis to the particle-hole channel, deriving the contribution to the continuity equation of the isoscalar and isovector s.e., v.c. and MEC particle-hole matrix elements. We start by evaluating the divergence of the correlation particle-hole matrix element $\left\langle p h^{-1}\left|\hat{j}_{\mu}^{C}\right| F\right\rangle$ for the s.e. and v.c. contributions; next we address the MEC ph matrix elements.

- $\underline{\text { Self energy }}$

From eqs. (25,26) we get

$$
\begin{gathered}
Q \cdot \mathcal{H}_{p}=-\frac{3 f^{2}}{2 m V^{2} m_{\pi}^{2}} \frac{m}{\sqrt{E_{\mathbf{p}} E_{\mathbf{h}}}} \sum_{\mathbf{k}} \frac{m}{E_{\mathbf{k}}} \bar{u}(\mathbf{p}) \frac{(P-\not K)(K-m)(\not-\not K)}{(P-K)^{2}-m_{\pi}^{2}} S_{F}(P) F_{1} \not P u(\mathbf{h}) \\
Q \cdot \mathcal{H}_{h}=-\frac{3 f^{2}}{2 m V^{2} m_{\pi}^{2}} \frac{m}{\sqrt{E_{\mathbf{p}} E_{\mathbf{h}}}} \sum_{\mathbf{k}} \frac{m}{E_{\mathbf{k}}} \bar{u}(\mathbf{p}) F_{1} \not Q S_{F}(H) \frac{(K-\not H)(K-m)(K-\not H)}{(K-H)^{2}-m_{\pi}^{2}} u(\mathbf{h}) .
\end{gathered}
$$

Note that $F_{1}$ cannot be taken out of the matrix element since it acts on the isospinors. Now from the relations

$$
\begin{aligned}
S_{F}(P) \varnothing u(\mathbf{h}) & =u(\mathbf{h}) \\
\bar{u}(\mathbf{p}) \not \subset S_{F}(H) & =-\bar{u}(\mathbf{p}) \\
\bar{u}(\mathbf{p})(\not P-\not K)(\not K-m) & =2 m \bar{u}(\mathbf{p})(\not P-\not K) \\
(\not K-m)(K-\not H) u(\mathbf{h}) & =-2 m(K-m) u(\mathbf{h})
\end{aligned}
$$

the following expressions are derived:

$$
Q \cdot \mathcal{H}_{p}=-\frac{3 f^{2}}{V^{2} m_{\pi}^{2}} \frac{m}{\sqrt{E_{\mathbf{p}} E_{\mathbf{h}}}} \sum_{\mathbf{k}} \frac{m}{E_{\mathbf{k}}} \bar{u}(\mathbf{p}) \frac{(K-m)(P-\not K)}{(P-K)^{2}-m_{\pi}^{2}} F_{1} u(\mathbf{h})
$$




$$
Q \cdot \mathcal{H}_{h}=-\frac{3 f^{2}}{V^{2} m_{\pi}^{2}} \frac{m}{\sqrt{E_{\mathbf{p}} E_{\mathbf{h}}}} \sum_{\mathbf{k}} \frac{m}{E_{\mathbf{k}}} \bar{u}(\mathbf{p}) F_{1} \frac{(K-\not H)(K-m)}{(K-H)^{2}-m_{\pi}^{2}} u(\mathbf{h})
$$

- $\underline{\text { Vertex correlations }}$

From eqs. (27,28) the four-divergence of the v.c. matrix element is found to be

$$
\begin{aligned}
& Q \cdot \mathcal{F}=-\frac{f^{2}}{V^{2} m_{\pi}^{2}} \frac{m}{\sqrt{E_{\mathbf{p}} E_{\mathbf{h}}}} \sum_{\mathbf{k}} \frac{m}{E_{\mathbf{k}}} \bar{u}(\mathbf{p}) \gamma_{5}(K-\not H) S_{F}(K+Q) \tau_{a} F_{1} \tau_{a} \varnothing \gamma_{5} \frac{\not K-m}{(K-H)^{2}-m_{\pi}^{2}} u(\mathbf{h}) \\
& Q \cdot \mathcal{B}=-\frac{f^{2}}{V^{2} m_{\pi}^{2}} \frac{m}{\sqrt{E_{\mathbf{p}} E_{\mathbf{h}}}} \sum_{\mathbf{k}} \frac{m}{E_{\mathbf{k}}} \bar{u}(\mathbf{p}) \frac{K-m}{(P-K)^{2}-m_{\pi}^{2}} \tau_{a} F_{1} \tau_{a} \gamma_{5} \varnothing S_{F}(K-Q) \gamma_{5}(\not P-\not K) u(\mathbf{h}) .
\end{aligned}
$$

We now exploit the identities

$$
\begin{aligned}
& S_{F}(K+Q) \varnothing(K+m)=+(K+m) \\
& (K+m) \varnothing S_{F}(K-Q)=-(K+m)
\end{aligned}
$$

to get finally

$$
\begin{aligned}
& Q \cdot \mathcal{F}=\frac{f^{2}}{V^{2} m_{\pi}^{2}} \frac{m}{\sqrt{E_{\mathbf{p}} E_{\mathbf{h}}}} \sum_{\mathbf{k}} \frac{m}{E_{\mathbf{k}}} \bar{u}(\mathbf{p}) \tau_{a} F_{1} \tau_{a} \frac{(K-\not H)(K-m)}{(K-H)^{2}-m_{\pi}^{2}} u(\mathbf{h}) \\
& Q \cdot \mathcal{B}=\frac{f^{2}}{V^{2} m_{\pi}^{2}} \frac{m}{\sqrt{E_{\mathbf{p}} E_{\mathbf{h}}}} \sum_{\mathbf{k}} \frac{m}{E_{\mathbf{k}}} \bar{u}(\mathbf{p}) \tau_{a} F_{1} \tau_{a} \frac{(K-m)(\not P-\not K)}{(P-K)^{2}-m_{\pi}^{2}} u(\mathbf{h}) .
\end{aligned}
$$

If the expressions (62, 63, 66, 67) are split into their isoscalar and isovector parts, as illustrated in Section 3, we get

$$
\begin{aligned}
Q \cdot \mathcal{H}_{p}^{(S)} & =-\frac{3 f^{2}}{V^{2} m_{\pi}^{2}} F_{1}^{(S)} \frac{m}{\sqrt{E_{\mathbf{p}} E_{\mathbf{h}}}} \sum_{\mathbf{k}} \frac{m}{E_{\mathbf{k}}} \bar{u}(\mathbf{p}) \frac{(K-m)(P-\not K)}{(P-K)^{2}-m_{\pi}^{2}} u(\mathbf{h}) \\
Q \cdot \mathcal{H}_{p}^{(V)} & =-\frac{3 f^{2}}{V^{2} m_{\pi}^{2}} F_{1}^{(V)} \frac{m}{\sqrt{E_{\mathbf{p}} E_{\mathbf{h}}}} \sum_{\mathbf{k}} \frac{m}{E_{\mathbf{k}}} \bar{u}(\mathbf{p}) \frac{(K-m)(\not P-\not K)}{(P-K)^{2}-m_{\pi}^{2}} u(\mathbf{h}) \\
Q \cdot \mathcal{H}_{h}^{(S)} & =-\frac{3 f^{2}}{V^{2} m_{\pi}^{2}} F_{1}^{(S)} \frac{m}{\sqrt{E_{\mathbf{p}} E_{\mathbf{h}}}} \sum_{\mathbf{k}} \frac{m}{E_{\mathbf{k}}} \bar{u}(\mathbf{p}) \frac{(K-\not H)(K-m)}{(K-H)^{2}-m_{\pi}^{2}} u(\mathbf{h}) \\
Q \cdot \mathcal{H}_{h}^{(V)} & =-\frac{3 f^{2}}{V^{2} m_{\pi}^{2}} F_{1}^{(V)} \frac{m}{\sqrt{E_{\mathbf{p}} E_{\mathbf{h}}}} \sum_{\mathbf{k}} \frac{m}{E_{\mathbf{k}}} \bar{u}(\mathbf{p}) \frac{(K-\not H)(K-m) \tau_{3}}{(K-H)^{2}-m_{\pi}^{2}} u(\mathbf{h}) \\
Q \cdot \mathcal{F}^{(S)} & =+\frac{3 f^{2}}{V^{2} m_{\pi}^{2}} F_{1}^{(S)} \frac{m}{\sqrt{E_{\mathbf{p}} E_{\mathbf{h}}}} \sum_{\mathbf{k}} \frac{m}{E_{\mathbf{k}}} \bar{u}(\mathbf{p}) \frac{(K-\not H)\left(K-m^{2}\right)}{(K-H)^{2}-m_{\pi}^{2}} u(\mathbf{h}) \\
Q \cdot \mathcal{F}^{(V)} & =+\frac{f^{2}}{V^{2} m_{\pi}^{2}} F_{1}^{(V)} \frac{m}{\sqrt{E_{\mathbf{p}} E_{\mathbf{h}}}} \sum_{\mathbf{k}} \frac{m}{E_{\mathbf{k}}} \bar{u}(\mathbf{p}) \frac{(K-\not H)\left(K-m_{)}\right.}{(K-H)^{2}-m_{\pi}^{2}}\left(\tau_{3}+i \varepsilon_{3 a b} \tau_{a} \tau_{b}\right) u(\mathbf{h})
\end{aligned}
$$




$$
\begin{aligned}
Q \cdot \mathcal{B}^{(S)} & =+\frac{3 f^{2}}{V^{2} m_{\pi}^{2}} F_{1}^{(S)} \frac{m}{\sqrt{E_{\mathbf{p}} E_{\mathbf{h}}}} \sum_{\mathbf{k}} \frac{m}{E_{\mathbf{k}}} \bar{u}(\mathbf{p}) \frac{(K-m)(P-\not K)}{(P-K)^{2}-m_{\pi}^{2}} u(\mathbf{h}) \\
Q \cdot \mathcal{B}^{(V)} & =+\frac{f^{2}}{V^{2} m_{\pi}^{2}} F_{1}^{(V)} \frac{m}{\sqrt{E_{\mathbf{p}} E_{\mathbf{h}}}} \sum_{\mathbf{k}} \frac{m}{E_{\mathbf{k}}} \bar{u}(\mathbf{p}) \frac{(K-m)(P-\not K)}{(P-K)^{2}-m_{\pi}^{2}}\left(\tau_{3}+i \varepsilon_{3 a b} \tau_{a} \tau_{b}\right) u(\mathbf{h}) .
\end{aligned}
$$

From these relations we learn that:

- In the isoscalar channel the self-energy and vertex contributions cancel

$$
Q \cdot \mathcal{H}_{p}^{(S)}+Q \cdot \mathcal{B}^{(S)}=Q \cdot \mathcal{H}_{h}^{(S)}+Q \cdot \mathcal{F}^{(S)}=0 .
$$

This is in contrast with the non-relativistic result [12], where the self-energy is by itself gauge invariant.

- In the isovector channel we get

$$
\begin{aligned}
Q \cdot\left[\mathcal{H}_{p}^{(V)}+\mathcal{B}^{(V)}\right] & =\frac{2 f^{2}}{V^{2} m_{\pi}^{2}} F_{1}^{(V)} i \varepsilon_{3 a b} \frac{m}{\sqrt{E_{\mathbf{p}} E_{\mathbf{h}}}} \sum_{\mathbf{k}} \frac{m}{E_{\mathbf{k}}} \bar{u}(\mathbf{p}) \frac{(K-m)(\not P-\not K) \tau_{a} \tau_{b}}{(P-K)^{2}-m_{\pi}^{2}} u(\mathbf{h}) \\
Q \cdot\left[\mathcal{H}_{h}^{(V)}+\mathcal{F}^{(V)}\right] & =\frac{2 f^{2}}{V^{2} m_{\pi}^{2}} F_{1}^{(V)} i \varepsilon_{3 a b} \frac{m}{\sqrt{E_{\mathbf{p}} E_{\mathbf{h}}}} \sum_{\mathbf{k}} \frac{m}{E_{\mathbf{k}}} \bar{u}(\mathbf{p}) \frac{(K-\not H)(K-m) \tau_{a} \tau_{b}}{(K-H)^{2}-m_{\pi}^{2}} u(\mathbf{h}) .
\end{aligned}
$$

These expressions, using the Dirac equations $H u(\mathbf{h})=m u(\mathbf{h})$ and $\bar{u}(\mathbf{p}) \not P=m \bar{u}(\mathbf{p})$, can be further simplified to yield the following four-divergence of the correlation current

$$
\begin{aligned}
& \left\langle Q \cdot \hat{j}^{C}\right\rangle=\frac{1}{2} Q \cdot\left[\mathcal{H}_{p}^{(V)}+\mathcal{B}^{(V)}+\mathcal{H}_{h}^{(V)}+\mathcal{F}^{(V)}\right] \\
= & \frac{2 f^{2}}{V^{2} m_{\pi}^{2}} F_{1}^{(V)} i \varepsilon_{3 a b} \frac{m}{\sqrt{E_{\mathbf{p}} E_{\mathbf{h}}}} \sum_{\mathbf{k}} \frac{m}{E_{\mathbf{k}}} \bar{u}(\mathbf{p}) \tau_{a}\left\{\frac{K \cdot P-m^{2}}{(P-K)^{2}-m_{\pi}^{2}}-\frac{K \cdot H-m^{2}}{(K-H)^{2}-m_{\pi}^{2}}\right\} \tau_{b} u(\mathbf{h}) .
\end{aligned}
$$

This contribution is exactly cancelled by that of the MEC (seagull and pion-in-flight) as we illustrate in what follows.

- $\underline{\mathrm{MEC}}$

Using the expressions given in Section 3.2 for the ph matrix elements corresponding to the seagull and pion-in-flight currents in eqs. (21,22), the associated four-divergences are found to be

$$
\begin{aligned}
\left\langle Q \cdot \hat{j}^{S}\right\rangle & =-\frac{f^{2}}{V^{2} m_{\pi}^{2}} F_{1}^{(V)} i \varepsilon_{3 a b} \frac{m}{\sqrt{E_{\mathbf{p}} E_{\mathbf{h}}}} \\
& \times \sum_{\mathbf{k}} \frac{m}{E_{\mathbf{k}}} \bar{u}(\mathbf{p}) \tau_{a} \tau_{b}\left\{\frac{(K-m) \varnothing}{(P-K)^{2}-m_{\pi}^{2}}+\frac{\varnothing(K-m)}{(K-H)^{2}-m_{\pi}^{2}}\right\} u(\mathbf{h}) \\
\left\langle Q \cdot \hat{j}^{P}\right\rangle & =\frac{2 m f^{2}}{V^{2} m_{\pi}^{2}} F_{1}^{(V)} i \varepsilon_{3 a b} \frac{m}{\sqrt{E_{\mathbf{p}} E_{\mathbf{h}}}} \\
& \times \sum_{\mathbf{k}} \frac{m}{E_{\mathbf{k}}} \frac{\left(Q^{2}+2 H \cdot Q-2 K \cdot Q\right)}{\left[(P-K)^{2}-m_{\pi}^{2}\right]\left[(K-H)^{2}-m_{\pi}^{2}\right]} \bar{u}(\mathbf{p}) \tau_{a}(K-m) \tau_{b} u(\mathbf{h}) .
\end{aligned}
$$


Exploiting the Dirac equation and after some algebra the above can be recast as follows

$$
\begin{aligned}
& \left\langle Q \cdot \hat{j}^{M E C}\right\rangle=\left\langle Q \cdot \hat{j}^{S}\right\rangle+\left\langle Q \cdot \hat{j}^{P}\right\rangle \\
= & -\frac{2 f^{2}}{V^{2} m_{\pi}^{2}} F_{1}^{(V)} i \varepsilon_{3 a b} \frac{m}{\sqrt{E_{\mathbf{p}} E_{\mathbf{h}}}} \sum_{\mathbf{k}} \frac{m}{E_{\mathbf{k}}} \bar{u}(\mathbf{p}) \tau_{a}\left\{\frac{K \cdot P-m^{2}}{(P-K)^{2}-m_{\pi}^{2}}-\frac{K \cdot H-m^{2}}{(K-H)^{2}-m_{\pi}^{2}}\right\} \tau_{b} u(\mathbf{h}) .
\end{aligned}
$$

We have thus proven that the correlation and MEC ph matrix elements satisfy current conservation, i.e., $\left\langle Q \cdot \hat{j}^{C}\right\rangle+\left\langle Q \cdot \hat{j}^{M E C}\right\rangle=0$.

\section{Appendix B. Poles in the vertex correlation diagrams}

- Forward Diagram

As shown in Section 3.2, the forward-going diagram (Fig. 2d) of the v.c. ph matrix element involves integrals of the type

$$
\int d \mathbf{k} \frac{f(\mathbf{k})}{(K+Q)^{2}-m^{2}+i \epsilon}
$$

The question then is if, for given $q$ and $\omega$, a four-momentum $K^{\mu}$, with $k<k_{F}$, exists such that $P^{\prime}=K+Q$ is on-shell. The three-momentum $\mathbf{k}$ lies on the sphere $A$ with radius $k$ (see Fig. 21). On the other hand the three-momentum $\mathbf{p}^{\prime}$ should lie on the sphere $C$ of radius

$$
p^{\prime}=\sqrt{\left(E_{\mathbf{k}}+\omega\right)^{2}-m^{2}}
$$

where $E_{\mathbf{k}}=\sqrt{k^{2}+m^{2}}$ is the energy of the hole. Moreover, since $\mathbf{p}^{\prime}=\mathbf{k}+\mathbf{q}$, it should also lie on the sphere $B$ which corresponds to $A$ translated by an amount $\mathbf{q}$. The pole is clearly hit when the two spheres $B$ and $C$ intersect.

A quantitative assessment of the position of the poles can be more easily derived in the non-relativistic framework. In this case indeed the particle momentum reads

$$
p^{2}=k^{2}+2 m \omega .
$$

Let us now keep $q$ and $k$ fixed and study the behaviour of the poles as a function of $\omega$. On the basis of eq. (71) we can consider three situations:

- i) $\omega=q^{2} / 2 m(\mathrm{QEP})$. Here $p^{\prime 2}=k^{2}+q^{2}$ and consequently $\mathbf{k}$ is perpendicular to $\mathbf{q}$, so the pole appears for $\cos \theta_{k}=0$. This situation is illustrated in Fig. 21(b).

- ii) $\omega>q^{2} / 2 m$ (above QEP). The particle momentum satisfies $p^{2}>k^{2}+q^{2}$ (Fig. 21(a)). In this situation the radius of the sphere $C$ is larger than in the previous case (Fig. 21(a)), and therefore $\theta_{k}<\frac{\pi}{2}$ and $\cos \theta_{k}>0$.

- iii) $\omega<q^{2} / 2 m$ (below QEP), i.e., $p^{2}<k^{2}+q^{2}$ (Fig. 21(c)). Here $\theta_{k}>\frac{\pi}{2}$ and $\cos \theta_{k}<0$. 


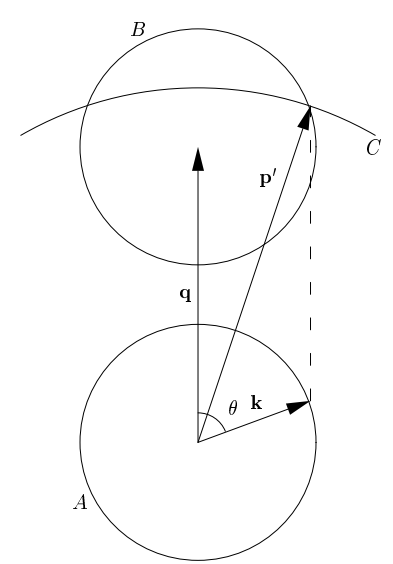

(a)

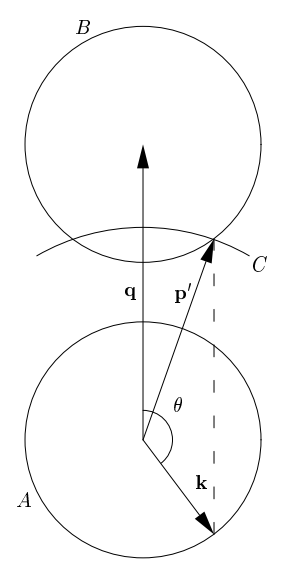

(c)

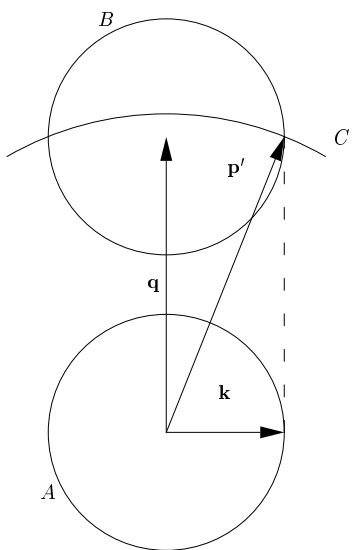

(b)

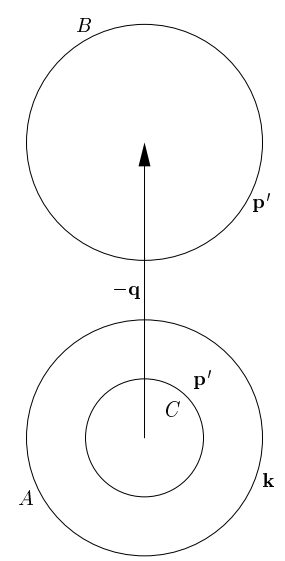

(d)

Figure 21: Poles in vertex correlation. Diagrams (a-c) refer to the forward-going situation and (d) to the backward-going one.

To estimate the minimum value of the hole momentum $k$ allowing for the existence of a pole, from Figs. 21(a) and 21(c) it clearly appears that, for given $q$ and $\omega$, there are no poles if the value of $k$ is very small: in fact in this case the spheres $B$ and $C$ do not intersect. More precisely, the range of allowed values of $k$ is limited by the condition (for $k<q$ ):

$$
q-k<p^{\prime}<q+k .
$$

Within the non-relativistic framework (see eq. (71)), this condition reads

$$
|m \Delta \omega|<q k
$$

where $\Delta \omega$ is defined as the $\omega$-shift from the QEP: $\Delta \omega \equiv \omega-\frac{q^{2}}{2 m}$. Then the minumum value of $k$ allowing for the existence of a pole is

$$
k_{\min }=\frac{m}{q}\left|\omega-\frac{q^{2}}{2 m}\right|=m\left|\frac{\lambda}{\kappa}-\kappa\right| .
$$


It then follows that at the QEP one has $k_{\min }=0$ and therefore the pole always comes into play.

- $\underline{\text { Backward Diagram }}$

In the case of the backward-going v.c. (Fig. 21(d)) the integral to be considered is

$$
\int d \mathbf{k} \frac{g(\mathbf{k})}{(K-Q)^{2}-m^{2}+i \epsilon} .
$$

Also here one can study the conditions that allow a particle with four-momenutm $P^{\prime} \equiv K-Q$ to be on-shell for given $q$ and $\omega$. Now clearly the energy associated with $p^{\prime}$ is $E_{\mathbf{p}}^{\prime}=E_{\mathbf{k}}-\omega$ with $E_{\mathbf{k}}=\sqrt{k^{2}+m^{2}}$. The momentum $p^{\prime}$ must lie on the sphere $C$ of radius

$$
p^{\prime}=\sqrt{\left(E_{\mathbf{k}}-\omega\right)^{2}-m^{2}}<k<k_{F},
$$

which now is contained inside the sphere $A$ (see Fig. 21(d)). On the other hand, since $\mathbf{p}^{\prime}=\mathbf{k}-\mathbf{q}$, it must also lie on the sphere $B$ obtained for $A$ by translating by an amount $-\mathbf{q}$. For $q>2 k_{F}$ (no Pauli-blocking) the intersection between $B$ and $C$ vanishes: hence no poles can be found.

\section{Appendix C. Polarization propagator with nucleon self-energy}

In this appendix we derive the general expression for the self-energy polarization propagator with any number of insertions, $\Sigma(P)$, in the particle and/or in the hole lines. From the obtained expression one can derive, as particular cases, the leading-order response (no interaction lines) and the first-order self-energy response (with one interaction line).

Let us then consider the polarization propagator containing $n$ self-energy insertions $\Sigma(H)$ in the hole line and $l$ insertions $\Sigma(P)$ in the particle line. It is given by

$$
\Pi^{\mu \nu}=-i \operatorname{Tr} \int \frac{d h_{0} d^{3} h}{(2 \pi)^{4}} \Gamma^{\mu}(Q)[S(H) \Sigma(H)]^{n} S(H) \Gamma^{\nu}(-Q)[S(H+Q) \Sigma(H+Q)]^{l} S(H+Q) .
$$

Using the nucleon propagator in the medium in eq. (42) we can express the product of $n+1$ propagators appearing in eq. (77) as a derivative of order $n$ according to

$$
\begin{aligned}
{[S(H)} & \Sigma(H)]^{n} S(H)= \\
& =[(H+m) \Sigma(H)]^{n}(\not H+m)\left[\frac{\theta\left(h-k_{F}\right)}{\left(H^{2}-m^{2}+i \epsilon\right)^{n+1}}+\frac{\theta\left(k_{F}-h\right)}{\left(H^{2}-m^{2}-i \epsilon h_{0}\right)^{n+1}}\right] \\
& =\left.[(H+m) \Sigma(H)]^{n}(\not H+m) \frac{1}{n !} \frac{d^{n}}{d \alpha^{n}}\right|_{\alpha=0}\left[\frac{\theta\left(h-k_{F}\right)}{H^{2}-\alpha-m^{2}+i \epsilon}+\frac{\theta\left(k_{F}-h\right)}{H^{2}-\alpha-m^{2}-i \epsilon h_{0}}\right] \\
& =[(H+m) \Sigma(H)]^{n}(\not H+m) \\
& \times\left.\frac{1}{n !} \frac{d^{n}}{d \alpha^{n}}\right|_{\alpha=0}\left[\frac{1}{H^{2}-\alpha-m^{2}+i \epsilon}+2 \pi i \theta\left(k_{F}-h\right) \delta\left(H^{2}-\alpha-m^{2}\right) \theta\left(h_{0}\right)\right] .
\end{aligned}
$$


A similar equation holds for the propagation of a particle. The global polarization propagator, with both the particle and the hole line dressed, can then be written as

$$
\begin{aligned}
\Pi^{\mu \nu}= & -\left.\left.i \frac{1}{n ! l !} \frac{d^{n}}{d \alpha^{n}}\right|_{\alpha=0} \frac{d^{l}}{d \beta^{l}}\right|_{\beta=0} \\
& \times \operatorname{Tr} \int \frac{d h_{0} d^{3} h}{(2 \pi)^{4}} \Gamma^{\mu}(Q)[(\not H+m) \Sigma(H)]^{n}(\not H+m) \Gamma^{\nu}(-q)[(P+m) \Sigma(P)]^{l}(\not P+m) \\
& \times\left[\frac{1}{H^{2}-\alpha-m^{2}+i \epsilon}+2 \pi i \theta\left(k_{F}-h\right) \delta\left(H^{2}-\alpha-m^{2}\right) \theta\left(h_{0}\right)\right] \\
& \times\left[\frac{1}{P^{2}-\beta-m^{2}+i \epsilon}+2 \pi i \theta\left(k_{F}-p\right) \delta\left(P^{2}-\beta-m^{2}\right) \theta\left(p_{0}\right)\right]
\end{aligned}
$$

where $P=H+Q$.

It gives rise to four terms. One of these contains the product of the two free propagators, namely

$$
\left(\frac{1}{H^{2}-\alpha-m^{2}+i \epsilon}\right)\left(\frac{1}{P^{2}-\beta-m^{2}+i \epsilon}\right),
$$

and yields the divergent vacuum contribution, which should be subracted out and pertains to a domain beyond nuclear physics. Then, after some algebra, one obtains

$$
\begin{aligned}
\Pi^{\mu \nu}- & \Pi_{0}^{\mu \nu}=\left.\left.2 \pi \frac{d^{n}}{d \alpha^{n}}\right|_{\alpha=0} \frac{d^{l}}{d \beta^{l}}\right|_{\beta=0} \int \frac{d h_{0} d^{3} h}{(2 \pi)^{4}} I_{n l}^{\mu \nu}(H, P, Q) \\
& \times\left[\frac{\theta\left(k_{F}-p\right) \delta\left(P^{2}-\beta-m^{2}\right) \theta\left(p_{0}\right)}{H^{2}-\alpha-m^{2}+i \epsilon}+\frac{\theta\left(k_{F}-h\right) \delta\left(H^{2}-\alpha-m^{2}\right) \theta\left(h_{0}\right)}{H^{2}-\beta-m^{2}+i \epsilon}\right. \\
& \left.+2 \pi i \theta\left(k_{F}-p\right) \theta\left(k_{F}-h\right) \delta\left(P^{2}-\beta-m^{2}\right) \delta\left(H^{2}-\alpha-m^{2}\right) \theta\left(P_{0}\right) \theta\left(H_{0}\right)\right],
\end{aligned}
$$

where the function

$$
\begin{aligned}
& I_{n l}^{\mu \nu}(H, P, Q)=I_{n l}^{\mu \nu}\left(h_{0}, \mathbf{h} ; p_{0}, \mathbf{p} ; q_{0}, \mathbf{q}\right) \\
\equiv & \frac{1}{n ! l !} \operatorname{Tr}\left[\Gamma^{\mu}(Q)[(\not H+m) \Sigma(H)]^{n}(\not H+m) \Gamma^{\nu}(-Q)[(P+m) \Sigma(P)]^{l}(P+m)\right]
\end{aligned}
$$

has been introduced. According to eq. (5) we then obtain for the nuclear tensor

$$
\begin{aligned}
& -\frac{V}{\pi} \operatorname{Im}\left[\Pi^{\mu \nu}-\Pi_{0}^{\mu \nu}\right] \\
= & \left.\left.2 \pi \frac{d^{n}}{d \alpha^{n}}\right|_{\alpha=0} \frac{d^{l}}{d \beta^{l}}\right|_{\beta=0} \int \frac{d h_{0} d^{3} h}{(2 \pi)^{4}} I_{n l}^{\mu \nu}(H, P, Q) \delta\left(P^{2}-\beta-m^{2}\right) \delta\left(H^{2}-\alpha-m^{2}\right) \\
& \times\left[\theta\left(k_{F}-p\right) \theta\left(p_{0}\right)+\theta\left(k_{F}-h\right) \theta\left(h_{0}\right)-2 \theta\left(k_{F}-p\right) \theta\left(k_{F}-h\right) \theta\left(p_{0}\right) \theta\left(h_{0}\right)\right] .
\end{aligned}
$$


Exploiting now the identity

$$
\begin{array}{r}
{\left[\theta\left(k_{F}-p\right) \theta\left(p_{0}\right)+\theta\left(k_{F}-h\right) \theta\left(h_{0}\right)-2 \theta\left(k_{F}-p\right) \theta\left(k_{F}-h\right) \theta\left(p_{0}\right) \theta\left(h_{0}\right)\right]} \\
=\theta\left(k_{F}-h\right) \theta\left(h_{0}\right)\left[1-\theta\left(k_{F}-p\right) \theta\left(p_{0}\right)\right]+\theta\left(k_{F}-p\right) \theta\left(p_{0}\right)\left[1-\theta\left(k_{F}-h\right) \theta\left(h_{0}\right)\right]
\end{array}
$$

we can recast the above according to

$$
-\frac{1}{\pi} \operatorname{Im}\left[\Pi^{\mu \nu}-\Pi_{0}^{\mu \nu}\right]=W_{+}^{\mu \nu}+W_{-}^{\mu \nu}
$$

where

$$
\begin{aligned}
W_{+}^{\mu \nu}(Q)=\left.\left.2 \pi \frac{d^{n}}{d \alpha^{n}}\right|_{\alpha=0} \frac{d^{l}}{d \beta^{l}}\right|_{\beta=0} \int \frac{d h_{0} d^{3} h}{(2 \pi)^{4}} I_{n l}^{\mu \nu}(H, P, Q) \delta\left(P^{2}-\beta-m^{2}\right) \delta\left(H^{2}-\alpha-m^{2}\right) \\
\\
\times \theta\left(k_{F}-h\right) \theta\left(h_{0}\right)\left[1-\theta\left(k_{F}-p\right) \theta\left(p_{0}\right)\right]
\end{aligned}
$$

and

$$
\begin{aligned}
W_{-}^{\mu \nu}(Q)=\left.\left.2 \pi \frac{d^{n}}{d \alpha^{n}}\right|_{\alpha=0} \frac{d^{l}}{d \beta^{l}}\right|_{\beta=0} \int \frac{d h_{0} d^{3} h}{(2 \pi)^{4}} I_{n l}^{\mu \nu}(H, P, Q) \delta\left(P^{2}-\beta-m^{2}\right) \delta\left(H^{2}-\alpha-m^{2}\right) \\
\\
\times \theta\left(k_{F}-p\right) \theta\left(p_{0}\right)\left[1-\theta\left(k_{F}-h\right) \theta\left(h_{0}\right)\right] .
\end{aligned}
$$

Now eq. (86) corresponds to the usual hadronic tensor for electron scattering, whereas eq. (87) corresponds to a process with negative energy and momentum transfer, hence it should be disregarded.

Next the integration with respect to $h_{0}$ can be explicitly performed by using the second of the $\delta$-functions appearing in eq. (86). After some algebra one then gets for the hadronic tensor the expression

$$
\begin{aligned}
W^{\mu \nu}= & \left.\left.\frac{d^{n}}{d \alpha^{n}}\right|_{\alpha=0} \frac{d^{l}}{d \beta^{l}}\right|_{\beta=0} \int \frac{d^{3} h}{(2 \pi)^{3}} \frac{I_{n l}^{\mu \nu}\left(E_{\mathbf{h}}^{\prime}(\alpha), \mathbf{h} ; E_{\mathbf{h}}^{\prime}(\alpha)+q_{0}, \mathbf{p} ; q\right)}{2 E_{\mathbf{h}}^{\prime}(\alpha)} \\
& \times \frac{\delta\left(E_{\mathbf{h}}^{\prime}(\alpha)+q_{0}-E_{\mathbf{p}}^{\prime}(\beta)\right)}{2 E_{\mathbf{p}}^{\prime}(\beta)} \theta\left(k_{F}-h\right) \theta\left(p-k_{F}\right),
\end{aligned}
$$

where

$$
\begin{aligned}
& E_{\mathbf{h}}^{\prime}(\alpha)=\sqrt{h^{2}+\alpha+m^{2}} \\
& E_{\mathbf{p}}^{\prime}(\beta)=\sqrt{p^{2}+\beta+m^{2}} .
\end{aligned}
$$

Finally, exploiting the first $\delta$-function in eq. (86), one can cast the response function in the form

$$
\begin{aligned}
W^{\mu \nu}= & \left.\left.\frac{d^{n}}{d \alpha^{n}}\right|_{\alpha=0} \frac{d^{l}}{d \beta^{l}}\right|_{\beta=0} \int \frac{d^{3} h}{(2 \pi)^{3}} \frac{I_{n l}^{\mu \nu}\left(E_{\mathbf{h}}^{\prime}(\alpha), \mathbf{h} ; E_{\mathbf{p}}^{\prime}(\beta), \mathbf{p} ; q\right)}{4 E_{h}^{\prime}(\alpha) E_{\mathbf{p}}^{\prime}(\beta)} \\
& \times \delta\left(E_{\mathbf{h}}^{\prime}(\alpha)+q_{0}-E_{\mathbf{p}}^{\prime}(\beta)\right) \theta\left(k_{F}-h\right) \theta\left(p-k_{F}\right) .
\end{aligned}
$$


For $n=l=0$ one gets the well known free hadronic tensor

$$
W^{\mu \nu}=\int \frac{d^{3} h}{(2 \pi)^{3}} \frac{I_{00}^{\mu \nu}\left(E_{\mathbf{h}}, \mathbf{h} ; E_{\mathbf{p}}, \mathbf{p} ; q\right)}{4 E_{\mathbf{h}} E_{\mathbf{p}}} \delta\left(E_{\mathbf{h}}+q_{0}-E_{\mathbf{p}}\right) \theta\left(k_{F}-h\right) \theta\left(p-k_{F}\right),
$$

for $n=1, l=0$ the first-order hole self-energy hadronic tensor

$$
W^{\mu \nu}=\left.\frac{d}{d \alpha}\right|_{\alpha=0} \int \frac{d^{3} h}{(2 \pi)^{3}} \frac{I_{10}^{\mu \nu}\left(E_{\mathbf{h}}^{\prime}(\alpha), \mathbf{h} ; E_{\mathbf{p}}, \mathbf{p} ; q\right)}{4 E_{\mathbf{h}}^{\prime}(\alpha) E_{\mathbf{p}}} \delta\left(E_{\mathbf{h}}^{\prime}(\alpha)+q_{0}-E_{\mathbf{p}}\right) \theta\left(k_{F}-h\right) \theta\left(p-k_{F}\right)
$$

and finally for $n=0, l=1$ the first-order particle self-energy hadronic tensor

$$
W^{\mu \nu}=\left.\frac{d}{d \beta}\right|_{\beta=0} \int \frac{d^{3} h}{(2 \pi)^{3}} \frac{I_{01}^{\mu \nu}\left(E_{\mathbf{h}}, \mathbf{h} ; E_{\mathbf{p}}^{\prime}(\beta), \mathbf{p} ; q\right)}{4 E_{\mathbf{h}} E_{\mathbf{p}}^{\prime}(\beta)} \delta\left(E_{\mathbf{h}}+q_{0}-E_{\mathbf{p}}^{\prime}(\beta)\right) \theta\left(k_{F}-h\right) \theta\left(p-k_{F}\right) .
$$

Note that the last two expressions coincide, as they should, with the ones already given in Section 4 .

The hadronic tensor in eq. (91) is one of the $(n+l)$-th order contributions to the full Hartree-Fock polarization propagator, which should be obtained as an infinite sum of all perturbative orders. It should be stressed that in a basically non-relativistic framework the HF series can be summed (see, e.g., [20]), whereas in our fully relativistic framework the summation is far more difficult to perform because of the matrix nature of the self-energy $\Sigma$ 36.

\section{References}

[1] E.J. Moniz et al., Phys. Rev. Lett. 46 (1971) 445.

[2] Z.E. Meziani et al., Phys. Rev. Lett. 52 (1984) 2130; 54 (1985) 1233.

[3] R. Altemus et al., Phys. Rev. Lett. 44 (1980) 965.

[4] P. Barreau et al., Nucl. Phys. A402 (1983) 515.

[5] G. Orlandini, M. Traini, Rep. Prog. Phys. 54 (1991) 257.

[6] M. Deady et al., Phys. Rev. C33 (1986) 1897.

[7] C.F. Williamson et al., Phys. Rev. C56 (1997) 3152.

[8] C.C. Blatchley et al., Phys. Rev. C34 (1986) 1243.

[9] J. Jourdan, Phys. Lett. B353 (1995) 189; Nucl. Phys. A603 (1996) 117.

[10] J. Dubach, J.H. Koch, T.W. Donnelly, Nucl. Phys. A271 (1976) 279; D.O. Riska, Phys. Rep. 181 (1989) 208; J.F. Mathiot, Phys. Rep. 173 (1989) 64. 
[11] J.W. Van Orden, T.W. Donnelly, Ann. Phys. 131 (1981) 451.

[12] W.M. Alberico, T.W. Donnelly, A. Molinari, Nucl. Phys. A512 (1990) 541.

[13] M.J. Dekker, P.J. Brussaard, J.A. Tjon, Phys. Rev. C49 (1994) 2650; Phys. Lett. B289 (1992) 255.

[14] J.E. Amaro, G. Co', E.M.V. Fasanelli, A.M. Lallena, Phys. Lett. B277 (1992) 249; J.E. Amaro, G. Co', A.M. Lallena, Ann. Phys. 221 (1993) 306.

[15] J.E. Amaro, G. Co', A.M. Lallena, Int. J. Mod. Phys. E3 (1994) 735.

[16] J.E. Amaro, G. Co', A.M. Lallena, Nucl. Phys. A578 (1994) 365.

[17] M. Kohno and N. Ohtsuka, Phys. Lett. B 98 (1981) 335.

[18] P.G. Blunden and M.N. Butler, Phys. Lett. B219 (1989) 151.

[19] J.E. Amaro, M.B. Barbaro, J.A. Caballero, T.W. Donnelly, A. Molinari, Nucl. Phys. A643 (1998) 349.

[20] W.M. Alberico, M.B. Barbaro, A. De Pace, T.W. Donnelly, A. Molinari, Nucl. Phys. A563 (1993) 605.

[21] M.B. Barbaro, A. De Pace, T.W. Donnelly, A. Molinari, Nucl. Phys. A569 (1994) 701.

[22] J.E. Amaro, M.B. Barbaro, J.A. Caballero, T.W. Donnelly, A. Molinari, Nucl. Phys. A657 (1999) 161.

[23] J.E. Amaro, M.B. Barbaro, J.A. Caballero, T.W. Donnelly, A. Molinari, in preparation (2001).

[24] T.W. Donnelly, Ingo Sick, Phys. Rev. Lett. 82 (1999) 3212 and Phys. Rev. C60:065502 (1999); W.M. Alberico, A. Molinari, T.W. Donnelly, E.L. Kronenberg, J.W. Van Orden, Phys. Rev. C38 (1988) 1801.

[25] T.W.Donnelly, A.S. Raskin, Ann. Phys. (N.Y.) 169 (1986) 247.

[26] T.W. Donnelly, M.J. Musolf, W.M. Alberico, M.B. Barbaro, A. De Pace, A. Molinari, Nucl. Phys. A541 (1992) 525.

[27] S. Boffi, C. Giusti, F.D. Pacati, Phys. Rep. 226 (1993) 1; S. Boffi, C. Giusti, F. Pacati, M. Radici, "Electromagnetic Response of Atomic Nuclei" (Oxford-Clarendon Press, 1996).

[28] M.B. Barbaro, A. De Pace, T.W. Donnelly and A. Molinari, "Electron scattering off nuclei" (Nova Science Publishing Co.).

[29] L. Alvarez-Ruso, M.B. Barbaro, T.W. Donnelly, A. Molinari, Phys. Lett. B497 (2001) 214. 
[30] C.R. Chinn, A. Picklesimer, J.W. Van Orden, Phys. Rev. C40 (1989) 790.

[31] A.L. Fetter, J.D. Walecka, "Quantum Theory of many-particle systems" (McGraw-Hill Publ. Co, 1971).

[32] M. Chemtob and M Rho, Nucl. Phys. A163 (1971) 1.

[33] S. Galster et al., Nucl. Phys. B32 (1971) 221.

[34] J.D. Bjorken, S.D. Drell, "Relativistic Quantum Mechanics" (McGraw-Hill, New York, 1964).

[35] E. Hadjimichael, G.I. Poulis, T.W. Donnelly, Phys. Rev. C45 (1992) 2666.

[36] B.D. Serot, J.D. Walecka, Adv. Nucl. Phys. 16 (1986) 1. 